\title{
RNA adenosine deaminase ADAR2 modulates T helper 17 cell effector function
}

\author{
Shengyun Ma ${ }^{1,4}$, Benjamin S. Cho ${ }^{1,4}$, Yuxin $\mathrm{Li}^{1}$, Nazia Abbasi ${ }^{1}$, Brian A. Yee ${ }^{1}$, Ge Sun ${ }^{1}$, \\ Claire Luo ${ }^{1}$, John T. Chang ${ }^{2}$, Bing Zhou ${ }^{1,3}$, Xiang-dong Fu ${ }^{1}$, Gene W. Yeo ${ }^{1}$, Wendy Jia \\ Men Huang ${ }^{1, *}$ \\ ${ }^{1}$ Department of Cellular and Molecular Medicine, \\ University of California San Diego, La Jolla, CA 92093, U.S.A \\ 2 Department of Medicine, \\ University of California San Diego, La Jolla, CA 92093, U.S.A \\ ${ }^{3}$ currently at Institute of Zoology Chinese Academy of Sciences, Beijing 100101, China \\ ${ }^{4}$ These authors contributed equally. \\ *Correspondence: wendyjmhuang@health.ucsd.edu
}




\section{SUMMARY}

Adenosine deaminases acting on RNA (ADARs) catalyze the most common RNA modification in mammals, but it remains to be elucidated how their RNA editing dependent and independent activities contribute to host immunity. Here, we report dynamic changes in ADARs expressions and global adenosine-to-inosin (A-to-I) editome during $T$ helper cell differentiation. In differentiated T helper 17 (Th17) cells, transcription of the ADAR2 encoding locus is potentiated by an intragenic super enhancer and splicing of the ADAR2 encoding transcript is further facilitated by a DEAD-box RNA helicase, DDX5. In an editing-independent manner, ADAR2 negatively regulates Hypoxia-Inducible Factor 1alpha (HIF1a) expression to limit the production of interleukin-10 (IL-10) in Th17 cells. These results demonstrate ADAR2 and the upstream mechanisms governing its expression as critical regulators of Th17 cell effector function. 


\section{INTRODUCTION}

Circulating naive $\mathrm{CD} 4^{+} \mathrm{T}$ lymphocytes encountering their cognate antigens differentiate into cytokine-producing effector cells to coordinate local and systemic immune responses (Reiner, 2009). The interleukin-17 (IL-17) producing effector cells are known as T-helper 17 (Th17). Th17 cells are heterogenous and can be functionally subdivided into two groups. The homeostatic subsets of Th17 cells secrete IL-10 and suppress inflammation in healthy hosts (Aschenbrenner et al., 2018; Bellemore et al., 2015; Chaudhry et al., 2011; Esplugues et al., 2011; McGeachy et al., 2007; Tanaka et al., 2018; Wu et al., 2018). In contrast, pathogenic subsets of Th17 cells contribute to autoimmune diseases and secrete a combination of proinflammatory cytokines, including IL-17, IL-21, and Granulocyte-macrophage colony-stimulating factor (GM-CSF) (Codarri et al., 2011; Stockinger and Omenetti, 2017; Tigno-Aranjuez et al., 2009). Paradoxically, while antibody blockades of Th17 cytokines benefit patients with psoriasis and arthritis (Yasuda et al., 2019), they worsen disease outcomes in patients with inflammatory bowel disease (Hueber et al., 2012). Better understanding of the molecular mechanisms underlying Th17 cytokine heterogeneity may provide improved therapeutic strategies to maintain homeostatic Th17 functions and also selectively block Th17 pathogenicity.

Adenosine deaminase acting on RNA (ADAR) is a highly conserved protein family that catalyzes the deamination of adenosine to inosine (A-to-I editing), the most abundant RNA modification in the mammalian transcriptome (Gallo et al., 2017; Tan et al., 2017) The ADAR family includes two active proteins, ADAR1 (encoded by Adar) and ADAR2 (encoded by Adarb1), as well as one catalytically inactive member, ADAR3 (encoded by 
Adarb2). ADAR1 and ADAR2 have shared and unique targets. They are unable to compensate for each other's essential functions in vivo (Chalk et al., 2019). ADAR1 primarily edits repetitive elements and prevents aberrant innate immune sensing of endogenous RNAs (Athanasiadis et al., 2004). ADAR2-mediated editing-dependent protein recoding of GRIA2 in neurons is essential for life (Higuchi et al., 2000). Importantly, ADARs also regulate other aspects of RNA biology independent of their catalytic activities. By binding to their target RNAs and interacting with other protein complexes, ADARs modulate alternative splicing, mRNA stability, microRNA biogenesis, and protein translation (Qi et al., 2017; Walkley and Li, 2017). In the immune system, ADAR1 in the thymus contribute to T cell maturation and self-tolerance (Nakahama et al., 2018). However, nothing is known about the editing dependent and independent roles of ADARs in mature T cells, including Th17.

Here, we report that naive and differentiated $\mathrm{CD}^{+} \mathrm{T}$ lymphocytes harbored unique and overlapping A-to-I editomes. Induction of ADAR2 in Th17 is driven transcriptionally by an intragenic super enhancer and post-transcriptionally by an RNA binding protein DDX5. Surprisingly, ADAR2 modulates effector cytokine production in Th17 cells in an editing independent manner. By blocking the pre-mRNA processing of Hif1a, ADAR2 impaired the expression of HIF1 $\alpha$ and its downstream target IL-10. Altered expression of ADAR2 or DDX5 in T cells result in dysregulated HIF $1 \alpha^{+} I L-10^{+}$Th17 population in the intestine. 


\section{RESULTS}

\section{Transcription regulation of Adarb1 in Th17 cells}

Total RNA-seq revealed that genes encoding different ADARs were dynamically expressed in $\mathrm{CD}^{+}$naive and differentiated Th17 cells. In particular, while Adar transcripts, encoding ADAR1, were downregulated in Th17 cells, Adarb1 transcripts, encoding ADAR2, were significantly upregulated (Figure 1A and 1B). Our observation is consistent with results from an independent RNA profile dataset (Figure S1A) (Stubbington et al., 2015). Western blot analyses confirmed that ADAR2 was abundantly expressed in Th17 cells (Figure 1C) and predominantly localized to the nucleus (Figure S2A).

Next, we sought to identify the cis-regulatory element(s) (CNS) and transcription factor(s) responsible for Adarb1 transcriptional activation during naive to Th17 polarization. Genome-wide characterization of acetylation on lysine 27 of histone H3 (H3K27ac) revealed a putative intragenic super-enhancer at the Adarb1 locus in Th17 cells (Figure 1D), containing four regions that opened up during naive to Th17 polarization (CNS1: chr10 77406713-77406733, CNS2: chr10 77388804-77388824, CNS3: chr10 7738787477387894, and CNS4: chr10 77368829-77368848) and one genomic region that was shut down in Th17 cells (CNS5: chr10 77367402-77367422) (Figure 1E). Time-course chromatin accessibility assay (ATAC-seq) revealed that chromatin openings at the CNS2, CNS3, and CNS4 regions closely correlate with the accessibility of Adarb1 promoter (Figure S3A), suggesting these CNSs may cooperate to drive Adarb1 transcription. To test this possibility, small guide RNAs (sgRNAs) designed to target each of the putative 
elements were introduced to Cas9-expressing Th17 cells. Targeting of the CNS2 and CNS3 regions resulted in significant reduction of Adarb1 mRNA expression (Figure 1F).

Analysis of a published transcription factor chromatin-immunoprecipitation (ChIP-seq) dataset (Ciofani et al., 2012) identified three Th17 master transcription factors, Basic Leucine Zipper ATF-Like Transcription Factor (BATF), Interferon Regulatory Factor 4 (IRF4), and RAR-related Orphan Receptor gamma (RORyt), were recruited to the Adarb1 super enhancer region in Th17 cells (Figure 1G). Analysis of a published transcriptomic dataset of Th17 cells obtained from BATF, IRF4, or RORyt knockout mice (Ciofani et al., 2012) revealed that Adarb1 expression was IRF4 dependent, and BATF and RORyt independent (Figure 1H). In summary, Adarb1 transcription in Th17 cells is facilitated by regional chromatin reorganization coupled to the establishment of an IRF4 occupying intragenic super enhancer.

\section{Naive and polarized Th17 cells have overlapping and distinct A-to-I RNA editomes}

ADARs catalyze RNA adenosine deamination. We hypothesized that the dynamic expression patterns of ADARs in naive and polarized Th17 cells would orchestrate distinct A-to-I editomes in these cells. To test this possibility, A-to-I edited transcripts from two independent biological replicates of naive and Th17 cells were identified using the Software for Accurately Identifying Locations of $\underline{R}$ AA-editing (SAILOR) (Washburn et al., 2014). With confidence scores greater than 0.99, we found 571 and 399 A-to-I edited sites, corresponding to transcripts from 270 and 131 genes, in naive and Th17 cells respectively (Figure 2A and Table S1). Majority of the edited sites localized to 3' 
untranslated regions (UTRs) (Figure 2B), similar to previous reports in other cell types (Athanasiadis et al., 2004; Qiu et al., 2016). Edited transcripts in naive and Th17 conditions had less than $18 \%$ overlap and encode for molecules enriched in different pathways, including $\mathrm{T}$ cell receptor signaling in naive cells and cytokine production in Th17 cells (Figure 2C and 2D).

\section{ADAR2 targets Hif1a transcripts to regulate Th17 effector cytokine production}

Several Th17 transcripts with A-to-I edits encode critical regulators of Th17 effector functions, including the Hypoxia-inducible factor alpha (HIF1a), C-C Chemokine Receptor 4 (CCR4), Interleukin-21 (IL-21), and Zinc finger and BTB domain containing 7B (ZBTB7B, also known as ThPok) (Figure 2E). Unlike most transcripts with A-to-I edits at the 3' UTR, the Hif1a transcript was edited at its $12^{\text {th }}$ intron. Immunoprecipitation using the antiADAR2 specific antibody suggested that ADAR2 associated with both the pre-processed and mature Hif1a transcript in Th17 cells (Figure S4A). Overexpression of wild type ADAR2, but not the enzymatically dead version (ADAR2 ${ }^{\mathrm{E} 396 \mathrm{~A}}$ ), enhanced the fraction of Hif1a transcripts harboring adenosine deamination (Figure $3 \mathrm{~A}$ and $3 \mathrm{~B}$ ). These results demonstrate that ADAR2 binds to and edits Hif1a transcripts in Th17 cells.

Next, we asked whether ADAR2 targets Hif1a transcript to regulate HIF1a expression. Intracellular protein staining revealed that cells overexpressing wild type ADAR2 had reduced HIF1a levels compared to control cells (Figure 3C and 3D). Surprisingly, overexpression of the enzymatically dead ADAR2 also dampened HIF1a protein levels. In ADAR2 ${ }^{\mathrm{E} 396 \mathrm{~A}}$-expressing Th17 cells, significant accumulation of unspliced Hif1a pre- 
mRNA was observed (Figure 3E and S5A). In contrast, CRISPR-Cas9 mediated knock down of ADAR2 lowered the fraction of Hif1a transcripts being edited and potentiated their proper splicing in Th17 cells (Figure 3F, 3G and 3H). These results highlight unexpected roles of ADAR2 in regulating Hif1a transcripts and protein expression through both enzymatic activity dependent and independent mechanisms.

HIF1a is a major regulator of cytokine IL-10 expression in B cells (Meng et al., 2018). Similarly, CRISPR-Cas9 mediated knock down of HIF1a also reduced IL-10 expression in Th17 cells (Figure 4A, 4B and 4C). Therefore, we hypothesized that negative regulation of HIF1 $\alpha$ expression by ADAR2 would affect IL-10 production in Th17 cells (modeled in Figure 4D). Indeed, overexpression of either wild type or mutant ADAR2 was sufficient to dampen IL-10 production in cultured Th17 cells (Figure 4E and 4F). To assess the role of ADAR2 in $\mathrm{CD}^{+} \mathrm{T}$ cells in vivo, activated $\mathrm{CD}^{+} \mathrm{T}$ cells transduced with ADAR2 ${ }^{\mathrm{WT}}$ or ADAR2 $^{\mathrm{E} 396 \mathrm{~A}}$ overexpression constructs were transferred into RAG1 knockout mice. Compared to empty vector transduced controls, recipients of either ADAR2 ${ }^{\mathrm{WT}}$ or ADAR2 ${ }^{\mathrm{E} 396 \mathrm{~A}}$ overexpressed cells experienced greater weight loss (Figure 4G), and RORyt $^{+}$Th17 cells recovered from their small intestine had reduced HIF1a and IL-10 expression (Figure 4H, 4I, and 4J). Interestingly, changes in ADAR2 expression did not significantly modulate HIF1a and IL-10 levels in the colon, suggesting ADAR2 likely regulates Th17 cell effector function in a tissue specific manner. In summary, these results demonstrate that ADAR2 negatively regulates HIF1 $\alpha$ and IL-10 expression in Th17 cells in vitro and in vivo. 


\section{Adarb1 pre-mRNA processing is regulated by DDX5 in Th17 cells}

Previous studies suggest that transcripts encoding proteins involved in RNA metabolism, such as Adarb1, are often regulated by other RNA binding proteins (RBP), including members of the DEAD-box (DDX) family of RNA helicases (Palladino et al., 2000). In Th17 cells, the most abundant transcript encoding DDXs is Ddx5 (Figure S6A) (Stubbington et al., 2015). Enhanced cross-linked immunoprecipitation analysis (eCLIPseq) using the DDX5-specific antibodies (Figure S6B) revealed several DDX5 footprints on the first intron of the Adarb1 transcript (Figure 5A). DDX5 can associate with heterogeneous nuclear ribonucleoprotein (hnRNP) and U1 small nuclear ribonucleoproteins to facilitate pre-mRNA splicing (Lin et al., 2005; Liu, 2002). To test the possibility that perhaps DDX5 binds to Adarb1 transcripts to regulate their posttranscriptional processing, wild type and DDX5-deficient Th17 cells were treated with flavopiridol, a transcription inhibitor, for eight hours and harvested to assess the ratio of spliced and unspliced Adarb1 transcripts as described in Figure S5. Loss of DDX5 resulted in a significant increase in the ratio of unspliced over spliced Adarb1 transcripts (Figure 5B). qRT-PCR and western blot analyses confirmed that DDX5 knocked out Th17 cells had lowered Adarb1 mRNA and ADAR2 protein (Figure 5C and 5D). Notably, RNAseq analyses of wild type and DDX5-deficient Th17 cells revealed that the expression of Irf4, encoding the transcription factor required for Adarb1 induction in Th17, was not affected in DDX5 knocked out Th17 cells (Figure S7A).

Unlike Adarb1, Adar transcripts did not carry any DDX5 footprint and mature Adar mRNA was upregulated in DDX5-deficient Th17 cells, which perhaps to compensate for the loss 
of Adarb1 expression (Figure S7B). We hypothesize that the imbalance of ADAR1 and ADAR2 expression in DDX5 knocked out Th17 cells may result in a dysregulated A-to-I editome. Indeed, SAILOR analyses of the transcriptome from two independent pairs of wild types and DDX5-deficient Th17 cells (Figure S8A and Table S2) uncovered a subset of Th17 transcripts harboring DDX5-dependent A-to-I edits (Figure 5E and Table S2), including Hif1a and the transcript encoding Apolipoprotein B Editing Complex 3 (Apobec3) (Figure S8B). PCR and Sanger sequencing of independent biological replicates confirmed significantly reduced fraction of these transcripts harboring A-to-I edits in DDX5-deficient Th17 cells (Figure 6A and S8C).

In the absence of DDX5, reduced ADAR2 levels and Hif1a editing were accompanied with an elevated expression of HIF1a and IL-10 (Figure 6B and 6C). CRISPR-Cas9 mediated mutation of a DDX5 bound region of Adarb1 promoted similar increases in HIF1 $\alpha$ and IL-10 (Figure 6D and 6E). In vivo, aberrant HIF1 $\alpha^{+} \mathrm{IL}-10^{+}$expressing Th17 cells were found in the small intestine from DDX5 T-cell specific knockout of mice (Figure $6 F)$. In summary, these results demonstrate that DDX5 regulates ADAR2 expression to fine-tune Th17 editome and effector cytokine production (modeled in Figure 6G).

\section{DISCUSSION}

ADARs not only catalyze RNA deamination, the most common RNA modification in mammals, they also had a number of catalytic activity-independent functions. For example, binding of ADAR1 to the Ctn RNA, independent of A-to-I editing, is sufficient to promote Ctn RNA stability by limiting the access of RNA decay complexes 
(Anantharaman et al., 2017). However, little is known about the molecular mechanisms governing ADAR expressions and their A-to-I editing dependent as well as independent functions in immune system. Identification and characterization of A-to-I edited transcripts in naive and Th17 cells uncovered the Hif1a transcript as a unique target of ADAR2 in Th17 cells. Unlike majority of A-to-I edited sites localizing to the 3'UTR, Hif1a is edited on intron 12 by ADAR2. Surprisingly, ADAR2 also negatively regulates Hif1a pre-mRNA processing in an enzymatic activity independent manner. We speculate that both passive and active mechanisms could be involved in this regulation. For instance, ADAR2 binding to Hif1a splicing junctions could directly limit the accessibility of the RNA transcript to the splicing machinery. ADAR2 may also recruit and/or cooperate with other negative regulators of splicing to form large protein complexes to interfere with Hif1a processing indirectly (Tang et al., 2020). Future studies will be needed to evaluate these possibilities.

HIF1a is a hypoxia inducible factor and plays important roles fine-tuning Th17 effector function in response to local tissue environment (Dang et al., 2011). Hypoxia has been known to promote IL-10 expression (Volchenkov et al., 2017). In CD1dhiCD5+ B cells, HIF1a promotes IL-10 expression to protect against autoimmunity (Meng et al., 2018). Similarly, here we report that HIF1a also promotes IL-10 expression in Th17 cells. Importantly, this pathway is regulated by ADAR2. Loss of ADAR2 expression results in an increase of HIF $1 \alpha$ and IL-10 levels in Th17 cells of the intestine.

ADARs expressions in naive and differentiated T cells are dynamically regulated. In Th17 cells, ADAR2 is induced in an IRF4 and intragenic super enhancer dependent manner. 
And processing of the Adarb1 pre-mRNAs is facilitated by an RNA-binding protein of the DEAD-box family, DDX5. Alterations in ADAR2 or DDX5 levels disrupt global A-to-I editing in Th17 cells and perturb the ability of homeostatic Th17 cell to make IL-10 cytokines in the small intestine. Intriguingly, colonic Th17 cells are not governed by the ADAR2-DDX5 axis. We speculate that this regional specificity is likely due to a combination of unique signaling molecules present in each microenvironment and the distinct repertoires of transcription factor expressed in Th17 cells from different tissues. Future studies will be needed to test whether increase of intestinal HIF $1 \alpha^{+} I L-10^{+}$Th17 cells in the T cell specific DDX5 knockout mice would alter host sensitivity to local inflammatory challenges, whether other DDX family members homologous to DDX5, such as DDX17, can compensate for the loss of DDX5 activities in disease settings, as well as whether the DDX5-ADAR2 mechanism described here can also contribute to regulating HIF1a-IL-10 expression in other cells types and in other tissue environments. Uncovering the roles of ADARs and A-to-I editing in $\mathrm{T}$ cells provide better understanding of the mechanisms regulating homeostatic Th17 effector functions and reveal new therapeutic targets for modulating their activities in health and disease. 


\section{ACKNOWLEDGEMENTS}

We thank Frances Fuller-Pace at the University of Dundee for sharing the DDX5 conditional mutant mice previously described in (Nicol et al., 2013). We thank Stefan Aigner, Alan Domissy, and Thai Bao Nguyen from the Gene Yeo Laboratory at the University of California San Diego for advice and technical support on the eCLIP-seq assays. We thank Johannes Zuber and Aichinger Martin at the Research Institute of Molecular Pathology (I.M.P.) for sharing the pSIN vector for generating retroviruses to carry custom sgRNAs into $\mathrm{T}$ cells. We thank Karen Sykes for suggestions to the manuscript. We thank Elina Zuniga and Li-Fan Lu for critiques and feedback to the project as members of the Master's thesis committee for B.S.C.

S.M., B.S.C., N.A., C.L. and W.J.M.H. are partially funded by the Edward Mallinckrodt, Jr. Foundation and the National Institutes of Health (NIH) (R01 GM124494 to WJM Huang). B.A.Y. and G.W.Y. are partially funded by NIH (R01 HG004659 and U41 HG009889 to GW Yeo). J.T.C. is partially funded by NIH (R01 Al129973). Illumina sequencing was conducted at the IGM Genomics Center, University of California San Diego, with support $\mathrm{NIH}$ (S10 OD026929) and Moores Cancer Center (P30 CA023100).

\section{AUTHOR CONTRIBUTIONS}

S.M. designed and performed ChIP-seq, ATAC-seq, RNA-seq, and flow cytometry studies. B.S.C. completed the Hif1a editing and Adarb1 splicing studies in Th17 cells with help from S.G. Y.L. performed the RNA-seq analyses (DESeq2, SAILOR, and Gene Ontology). N.A. designed and performed the eCLIP-seq experiment in Th17 cells. B.A.Y. 
performed bioinformatics analyses on the eCLIP-seq datasets. C.L. designed and performed sgRNA studies to assess enhancer activities of the Adarb1 regulatory elements. J.T.C. contributed resources and edited the manuscript. B.Z. performed the enhancer and super enhancer analyses on the ChIP-seq datasets. X.D.F. directed the enhancer analyses by B.Z. and edited the manuscript. G.W.Y directed analyses on the eCLIP-seq datasets and SAILOR and edited the manuscript. W.J.M.H. analyzed and wrote the manuscript together with S.M and B.C.

\section{DECLARATION OF INTERESTS}

GW Yeo is co-founder, member of the Board of Directors, on the Science Advisory Board, equity holder, and paid consultant for Locanabio and Eclipse Biolnnovations. GW Yeo is a visiting professor at the National University of Singapore. GW Yeo's interests have been reviewed and approved by the University of California San Diego in accordance with its conflict of interest policies. All other authors declare no competing financial interests. 


\section{FIGURE TITLES AND LEGENDS}

\section{Figure 1. An intragenic super enhancer promotes Adarb1 transcription in Th17 cells}

A. Volcano plot of RNA-seq results from $\mathrm{CD}^{+}$naive $(n=2)$ and cultured Th17 cells $(n=2)$. Black dots: differentially expressed genes defined as $\log _{2}$ fold change $\geq 1$ and $p$-value $<0.05$.

B. Heatmap of RNA expressions as determined by DEseq2 analysis of the RNA-seq dataset from A. Results from two independent biological replicates are shown.

C. Representative western blot analysis of whole cell extracts from $\mathrm{CD} 4^{+}$naive and Th17 cells. ADAR2 expression is quantified as signal of anti-ADAR2 antibody over those from the total protein stain. This experiment was repeated three times on independent biological samples with similar results.

D. Normalized histone H3 lysine 27 acetylation signals (RPM) in genomic regions within a $12.5 \mathrm{~Kb}$ window outside of any known-gene promoters (TSS $\pm 2.5 \mathrm{~Kb}$ ). Regions above the geometrically defined inflection point from this representation were considered putative super-enhancers (SE) and those below were consider putative traditional enhancers (TE) similar to previous report (Loven et al., 2013).

E. Integrative Genomic Viewer (IGV) displayed of the putative Adarb1 super enhancer identified in D. Chromatin accessibility of the locus was assessed by two independent ATAC-seq time course experiments on $\mathrm{CD} 4^{+}$naive T cells and Th17 cells harvested at 48 or 72 hours.

F. $\log _{2}$ fold changes in Adarb1 mRNA quantified by qRT-PCR in Cas9 ${ }^{+}$Th17 cells transduced with sgRNAs targeting the corresponding CNS compared to control. 
Average and standard deviation from two biological replicates are shown. * $p$ value $<0.05,{ }^{* *} p$-value $<0.01$ (t-test).

G. IGV display of BATF, IRF4, p300, and RORyt occupancies as determined by ChIPseq (GSE40918) (Ciofani et al., 2012) on the Adarb1 super enhancer.

H. Expression of Adarb1 in Th17 cell from WT, BATF, IRF4, and RORyt knockout mice as determined by RNA-seq (Ciofani et al., 2012). ${ }^{* * *} p$-value<0.001.

Figure 2. Overlapping and unique A-to-I editomes in wild type naive and Th17 cells

A. Fraction of transcripts harboring A-to-I edits as defined by SAILOR analysis on the RNA-seq datasets. Each dot represents transcripts from one gene. Linear regression line was used to calculate correlation score $\left(R^{2}\right)$ from two independent biological replicates of naive and Th17 cells.

B. Distribution of A-to-I edited sites on naive and Th17 transcripts from A.

C. The top 5 gene ontology pathways enriched in proteins encoded by A-to-I edited transcripts in naive and Th17 cells.

D. Shared and distinct A-to-I editomes in naive and Th17 cells.

E. RNA expression and \% A-to-I editing of Ccr4, Hif1a, II21, and Zbtb7b transcripts in naive and Th17 cells as determined by DEseq2 and SAILOR. Each dot represents result from one biological replicate (naive, $n=2 ;$ Th17, $n=2$ ).

\section{Figure 3. ADAR2 negatively regulates HIF1 $\alpha$ expression in Th17 cells}

A. Representative western blot analysis of whole cell extracts from Th17 cells transduced with empty, ADAR2 ${ }^{\mathrm{WT}}$, or enzymatically dead ADAR2 ${ }^{\mathrm{E} 396 \mathrm{~A}}$ expression 
constructs. This experiment was repeated two times on independent biological samples with similar results.

B. Representative RT-PCR and Sanger sequencing analyses on Hif1a transcripts from control, ADAR2 ${ }^{\mathrm{WT}}$, or enzymatically dead ADAR2 ${ }^{\mathrm{E} 396}$ overexpressed Th17 cells. Results are representative from two independent experiments.

C. Representative flow cytometry analysis of HIF1a and RORyt expression in Th17 cells transduced with control (black), ADAR2WT (red), or enzymatically dead ADAR2 $^{\mathrm{E} 396}$ (blue) expression constructs.

D. Summarized flow cytometry analysis results from three independent experiments described in C. * $p$-value $<0.05$ (paired t-test).

E. Ratio of unspliced to spliced Hif1a transcripts in control and ADAR2 ${ }^{\mathrm{E} 396}$ expressing Th17 cells as assessed by qRT-PCR. Each dot represents result from one biological replicate (control, $\mathrm{n}=4 ; \mathrm{ADAR} 2^{\mathrm{E396}}, \mathrm{n}=4$ ). * $p$-value $<0.05$ (paired t-test).

F. Representative western blot analysis of whole cell extracts from Cas9 ${ }^{+}$Th17 cells transduced with retrovirus carrying empty or sgRNA expression constructs designed to target the ATG translation start codon of Adarb1. This experiment was repeated three times on independent biological samples with similar results.

G. Representative RT-PCR and Sanger sequencing analyses on Hif1a transcripts from Th17 cells described in F, showing reduced A-to-I editing of Hif1a in ADAR2 knocked down cells. Results are representative from two independent experiments.

H. Ratio of unspliced to spliced Hif1a transcripts in control and ADAR2 knocked down Th17 cells as determined by qRT-PCR. * $p$-value<0.05 (t-test, $n=4)$. 
Figure 4. ADAR2-HIF1a regulates IL-10 production in Th17 cells in vitro and in vivo

A. Representative flow cytometry analysis of IL-10 and HIF $1 \alpha$ expression in Cas9+ Th17 cells transduced with control or Hif1a targeting sgRNA expression constructs. This experiment was repeated four times on independent biological samples with similar results.

B. Geometric mean fluorescent intensity (GMI) of HIF1a and RORyt in cells from experiments described in A. * $p$-value $<0.05$ (paired t-test, $n=4$ ).

C. Percentage of HIF1 $\alpha$ and/or IL-10 expressing in cells from experiments described in A. ${ }^{* *} p$-value<0.01 (paired t-test, $\mathrm{n}=4$ ).

D. Working model: ADAR2 targets Hif1a transcripts to negatively regulate HIF1a and its downstream target gene expression in Th17 cells.

E. Representative flow cytometry analysis of IL-10 expression in Th17 cells transduced with control, ADAR2 ${ }^{\mathrm{WT}}$, or enzymatically dead ADAR2 ${ }^{\mathrm{E} 396}$ expression constructs. This experiment was repeated three times on independent biological samples with similar results.

F. Summarized flow cytometry analysis results from independent experiments described in E. * $p$-value $<0.05$ and ${ }^{* *} p$-value $<0.01$ (paired t-test, $\mathrm{n}=3$ ).

G. Weight changes of Rag1 knockout mice receiving control $(n=6), \operatorname{ADAR}^{\mathrm{WT}}(\mathrm{n}=4)$, or enzymatically dead $\operatorname{ADAR2}^{\mathrm{E} 396}(\mathrm{n}=4)$ overexpressing $\mathrm{CD}^{+}{ }^{+}$T cells. ${ }^{*} p-$ value $<0.05$ and ${ }^{* *} p$-value $<0.01$ (Mann-Whitney test).

H. GMI of HIF1a in CD90.1 $1^{+} \mathrm{CD}^{+}$cells from the colonic and ileal lamina propria of the Rag1 knockout mice described in G on day 38. * $p$-value $<0.05$ (t-test). 
I. Proportion of RORyt ${ }^{+} \mathrm{HIF} 1 \alpha^{+} \mathrm{T}$ helper cells in colonic and ileal lamina propria harvested from recipient mice described in $\mathrm{G}$ on day 38 . * $p$-value $<0.05$ (t-test).

J. Proportion of $\mathrm{HIF} 1 \alpha^{+} \mathrm{IL}-10^{+} \mathrm{T}$ helper cells in colonic and ileum lamina propria harvested from recipient mice described in $\mathrm{G}$ on day 38 . * $p$-value $<0.05$ (t-test).

\section{Figure 5. Adarb1 is post-transcriptionally regulated by RNA binding protein DDX5}

A. Left: schematic representation of the DDX5 enhanced CLIP (eCLIP-seq) experiment. Right: IGV display of DDX5 eCLIP-seq signals on Adarb1 in Th17 cells.

B. Ratio of unspliced over spliced Adarb1 transcripts in $\mathrm{WT}^{\top}$ and DDX5 ${ }^{\Delta \mathrm{T}}$ Th17 cells treated with flavopiridol $(10 \mu \mathrm{M})$ for eight hours from three independent biological replicates. ${ }^{*} p$-value $<0.05$ (t-test).

C. Relative expression of mature Adarb1 mRNA as assessed by qRT-PCR from three independent biological replicates of $\mathrm{WT}^{\top}$ and DDX5 $5^{\Delta \mathrm{T}} \mathrm{Th} 17$ cells. Gapdh was used for normalization. ${ }^{* *} p$-value $<0.01$ (t-test).

D. Representative western blot analysis of whole cell extracts from $\mathrm{WT}^{\top}$ and $\mathrm{DDX} 5^{\Delta \mathrm{T}}$ Th17 cells. This experiment was repeated three times on independent biological samples with similar results.

E. Transcripts with DDX5-dependent and independent A-to-I edits as defined by SAILOR analysis of two independent biological replicates of $\mathrm{WT}^{\top}$ and $\mathrm{DDX} 5^{\Delta \mathrm{T}}$ Th17 cells. 


\section{Figure 6. DDX5 promotes HIF1a -IL-10 axis in vitro and in vivo}

A. Representative RT-PCR Sanger sequencing analysis of Hif1a transcripts in $\mathrm{WT}^{\top}$ and DDX5 $5^{\Delta \mathrm{T}}$ Th17 cells. This experiment was repeated two times on independent biological samples with similar results.

B. Representative flow cytometry analysis of HIF1 $\alpha$ and IL-10 expression in $\mathrm{WT}^{\top}$ and DDX5 $^{\Delta \mathrm{T}}$ Th17 cells polarized under IL-6 $(20 \mathrm{ng} / \mathrm{ml})$ and various TGF $\beta$ concentrations $(0-5.0 \mathrm{ng} / \mathrm{ml})$. This experiment was repeated three times on independent biological samples with similar results.

C. Summarized flow cytometry analysis results from three independent experiments described in B. * $p$-value $<0.05$ (t-test).

D. Representative flow cytometry analysis of RORyt, HIF1a, and IL-10 expression in Cas $9^{+}$Th17 cells transduced with empty or sgRNAs targeting DDX5 binding site (S3) on Adarb1. This experiment was repeated three times on independent biological samples with similar results.

E. Summarized flow cytometry analysis results from three independent experiments described in $\mathrm{D} .{ }^{*} p$-value $<0.05$ (paired t-test).

F. HIF1 $\alpha$ and IL-10 expression in $\mathrm{CD} 4^{+} \mathrm{T}$ cells from the distal small intestine (ileum) of $\mathrm{WT}^{\top}$ and $\mathrm{DDX} 5^{\Delta \mathrm{T}}$ mice. ${ }^{*} p$-value $<0.05$ (paired t-test, $\mathrm{n}=5$ ).

G. Working model: DDX5 and ADAR2 are negative regulators of the HIF1 $\alpha-I L-10$ axis in Th17 cells. 


\section{METHODS}

\section{Mice}

C57BL/6 wild-type (Stock No: 000664), Cas9 (Stock No: 026179), CD4Cre (Stock No: 017336), and Rag1 KO (Stock No: 002216) were obtained from the Jackson Laboratory. $D D X 5^{\text {flox }}$ mice were obtained from Dr. Frances Fuller-Pace's laboratory and have been previously described (Nicol et al., 2012). Heterozygous mice were bred to yield 6-8 weeks old $D d x 5^{+/+} C D 4 C r e^{+}$(subsequently referred to as wild type, $\mathrm{WT}^{\top}$ ) and $D d x 5^{\mathrm{fl/fl}} \mathrm{CD} 4 \mathrm{Cr} \mathrm{e}^{+}$ littermates (referred to as DDX5 ${ }^{\Delta \mathrm{T}}$ ). Adult mice at least eight weeks old were used. All animal studies were approved and followed the Institutional Animal Care and Use Guidelines of the University of California San Diego.

\section{Th17 Cell Culture}

Mouse naive T cells were purified from spleens and lymph nodes of 8-12 weeks old mice using the Naive CD4 ${ }^{+} \mathrm{T}$ Cell Isolation Kit according to the manufacturer's instructions (Miltenyi Biotec). Cells were cultured in Iscove's Modified Dulbecco's Medium (IMDM, Sigma Aldrich) and supplemented with 10\% heat-inactivated FBS (Peak Serum), $50 \mathrm{U}$ penicillin-streptomycin (Life Technologies), $2 \mathrm{mM}$ glutamine (Life Technologies), and 50 $\mu \mathrm{M} \beta$-mercaptoethanol (Sigma Aldrich). For polarized Th17 cell polarization, naive cells were seeded in 24-well or 96-well plates pre-coated with rabbit anti-hamster IgG and cultured in the presence of $0.25 \mu \mathrm{g} / \mathrm{mL}$ anti-CD3ع (eBioscience), $1 \mu \mathrm{g} / \mathrm{mL}$ anti-CD28 (eBioscience), $0.1-5.0 \mathrm{ng} / \mathrm{mL}$ TGF- $\beta$ (R\&D Systems), and $20 \mathrm{ng} / \mathrm{mL}$ IL-6 (R\&D Systems) for 48 to 72 hours. 


\section{Retrovirus Transduction in T cells}

sgRNAs were designed by CHOPCHOP (Labun et al., 2019)and the expressing retroviral constructs were generated as described previously (Ran et al., 2013). sgRNA sequences are listed in Table S3. Mouse ADAR2 overexpression constructs was generated by the In-Fusion ${ }^{\circledR}$ HD Cloning Plus kit (Takara). Specifically, primers were designed using Takara Primer Design tool with Notl and Sall extensions. The Adarb1 amplified PCR product was combined with the Notl and Sall linearized MSCV vector (RRID:Addgene_17442). ADAR2 ${ }^{\text {E396A }}$ expression construct was generated using the Q5 Site-Directed Mutagenesis Kit (NEB). Clones were screened and sequenced. Primers sequences are listed in Table S4.

PlatE cells were used to generate retrovirus as described (Morita et al., 2000). Virus transduction in T cells were performed 24 hours after T cell activation by centrifugation at $2000 \mathrm{rpm}$ for $90 \mathrm{~min}$ at $32{ }^{\circ} \mathrm{C}$. Live and Thy $1.1^{+}$retrovirus transduced cells cultured in Th17 polarizing conditions were analyzed by flow cytometry at $72 \mathrm{hrs}$.

\section{Flow Cytometry}

Cells were stimulated with $5 \mathrm{ng} / \mathrm{mL}$ Phorbol 12-myristate 13-acetate (PMA, Millipore Sigma) and $500 \mathrm{ng} / \mathrm{mL}$ ionomycin (Millipore Sigma) in the presence of GolgiStop (BD Bioscience) for 5 hours at $37^{\circ} \mathrm{C}$, followed by cell surface marker staining for CD4 (clone GK1.5; Biolegend) and CD90.1/Thy1.1 (clone HIS51; eBioscience). Fixation/Permeabilization buffers (eBioscience) were used as per manufacturer 
instructions to assess intracellular transcription factor and cytokine expression. Antibodies are listed in Table S5.

\section{RNA-seq}

Ribosome-depleted RNAs were used to prepare sequencing libraries. 100 bp paired-end sequencing was performed on an Illumina HiSeq4000 by the Institute of Genomic Medicine at the University of California San Diego. Each sample yielded approximately 30-40 million reads. Paired-end reads were aligned to the mouse mm10 genome with the STAR aligner version 2.6.1a (Dobin et al., 2013) using the parameters: "-outFilterMultimapNmax 20 --alignSJoverhangMin 8 --alignSJDBoverhangMin 1 -outFilterMismatchNmax 999 --outFilterMismatchNoverReadLmax 0.04 --alignIntronMin 20 --alignIntronMax 1000000 --alignMatesGapMax 1000000". Uniquely mapped reads overlapping with exons were counted using featureCounts (Liao et al., 2014) for each gene in the GENCODE.vM19 annotation. Differential expression analysis was performed using DESeq2 (v1.18.1 package) (Love et al., 2014), including a covariate in the design matrix to account for differences in harvest batch/time points. Regularized logarithm (rlog) transformation of the read counts of each gene was carried out using DESeq2. Pathway analysis was performed on differentially expressed protein coding genes with minimal counts of $10, \log _{2}$ fold change cutoffs of $\geq 0.5$ or $\leq-0.5$, and $p$-values $<0.05$ using Gene Ontology (http://www.geneontology.org/) where all expressed genes in the specific cell type were set as background. 
Transcripts harboring A-to-I edits in the RNA-seq datasets were identified by SAILOR version 1.0.4 in the paired-end mode (Washburn et al., 2014). Hits with confidence score less than 0.99 were filtered out. Known genomic variations were obtained from the Mouse Genomes Project. Variants were removed from the resulting candidate list if they overlapped with the known genomic variations. All remaining candidates were annotated based on GENCODE.vM19.

\section{ChIP-seq and ATAC-seq}

ChIP-seq were performed on 5-10 million Th17 cells crosslinked with $1 \%$ formaldehyde. Chromatin were sonicated and immunoprecipitated using antibodies listed on Table S5 and Dynabeads (ThermoFisher Scientific), followed by reverse cross-linking, and library construction as described in (Kaikkonen et al., 2013). ATAC-seq libraries were generated as described in (Buenrostro et al., 2015). ChIP-seq and ATAC-seq processing followed the ENCODE guideline with some modifications (Landt et al., 2012). Specifically, singleend raw reads were mapped to the mouse genome (GENCODE assembly GRCm38) by bowtie2 (Version 2.3.4.1) in the local mapping mode with parameter "--local", followed by PCR deduplication by SAMTools (Version 1.9) with the utility markedup (Li et al., 2009). Mapped reads from each sample repeats were merged into a single BAM file by SAMTools, and peaks were called using MACS2 (Version 2.2.6) (Zhang et al., 2008) in the narrow peak-calling mode with default parameters for ChIP-seq data or specific parameters of "callpeak --nomodel --extsize 100" for ATAC-seq data. Regions with peakscore below 30 were filtered out and the remaining reliable peak profiles were 
transformed into bigwig format and visualized on the Integrative Genomics Viewer (IGV Version 2.8.2) (Thorvaldsdottir et al., 2013).

\section{Identification of Enhancers and Super-enhancers}

Accessible chromatin regions away from known promoters (TSS $\pm 2.5 \mathrm{~kb}$ ) and overlapped with H3K27ac ChIP-seq peaks were defined as putative enhancers. Promoter-enhancer co-openness correlation score was calculated as previously described in (Zhou et al., 2019). To identify super-enhancers, H3K27ac peak regions within a $12.5 \mathrm{~Kb}$ window outside of any known-gene promoters (TSS $\pm 2.5 \mathrm{~Kb}$ ) were stitched together and ranked by increasing total background subtracted ChIP-seq occupancy of H3K27ac. Ranked regions were plotted against the total background subtracted ChIP-seq occupancy of H3K27ac in units of total rpm on the y-axis. Regions above the geometrically defined inflection point from this representation were considered putative super-enhancers similar to previous report (Loven et al., 2013).

\section{DDX5 eCLIP-seq and ADAR RIP}

eCLIP-seq analysis was performed as previously described (Van Nostrand et al., 2016). Primary cultured Th17 from two 8-10 weeks old wild-type (C57BL/6) female mice were subjected to UV-mediated crosslinking, lysis, and treatment with RNases, followed by immunoprecipitation (IP) of the DDX5-containing RNA complexes. RNA fragments protected from RNase digestion were subjected to RNA linker ligation, reversetranscription, and DNA linker ligation to generate eCLIP-seq libraries for high-throughput Illumina sequencing. Peaks were first defined using CLIPper on the IP sample 
(https://github.com/YeoLab/clipper/wiki/CLIPper-Home). Enrichments were calculated using both the IP and input samples. Log 2 fold change was calculated as eCLIP-seq reads normalized for read depth over normalized reads found at each peak region in the sizematched input sample. Significance tests were performed using the Fisher's exact test or chi squared test, as described (Van Nostrand et al., 2016). ENCODE Irreproducible Discovery Rate (IDR) analysis was performed on two independent biological replicates of Th17 cells. Peaks were ranked using the entropy formula, $\mathrm{Pi}^{*} \log (\mathrm{Pi} / \mathrm{Qi}) / \log _{2}$, where $\mathrm{Pi}$ is the probability of an eCLIP-seq read at that position and Qi is the probability of input reads at that position. Results were filtered using cutoffs of 3 for both $\log _{10} p$-values and $\log _{2}$ fold changes to define a set of true peaks normalized above their respective size-matched input background signal.

For ADAR RIP experiments, Th17 cells were lysed in $25 \mathrm{mM}$ Tris pH 8.0, $100 \mathrm{mM} \mathrm{NaCl}$, $0.5 \% \mathrm{NP} 40,10 \mathrm{mM} \mathrm{MgCl} 2,10 \%$ glycerol with RNase and protease inhibitors for $30 \mathrm{~min}$ on ice. Samples were spun down at $14,000 \times \mathrm{g}$ for $15 \mathrm{~min}$, and soluble protein lysates were harvested and immunoprecipitated with control or anti-ADAR2 specific antibody as listed on Table S5.

\section{Protein and Western Blot Analysis}

For whole cell lysates, cells were lysed in $25 \mathrm{mM}$ Tris pH 8.0, $100 \mathrm{mM} \mathrm{NaCl}, 0.5 \%$ NP40 with protease inhibitors for $30 \mathrm{~min}$ on ice. Samples were spun down at $14,000 \times g$ for 15 min, and soluble protein lysates were harvested. The NE-PER ${ }^{T M}$ kit (ThermoFisher Scientific) was used for cytoplasmic and nuclear fractionation studies. 30-50 $\mu$ g protein 
were loaded on each lane. Blots were blocked in Odyssey Blocking buffer (Li-CoR Biosciences) and probed with primary antibodies (Table S5). Following incubation with respective IRDye secondary antibody (Li-CoR Biosciences), infrared signals on each blot were measured on the Li-CoR Odyssey CLX.

\section{cDNA Synthesis, qRT-PCR, and RT-PCR Sanger Analysis}

Total RNA was extracted with the RNeasy kit (QIAGEN) and reverse transcribed using iScript $^{\mathrm{TM}}$ Select cDNA Synthesis Kit (Bio-Rad Laboratories). Real time RT-PCR was performed using $\mathrm{iTaq}^{\mathrm{TM}}$ Universal SYBR® Green Supermix (Bio-Rad Laboratories). Expression data was normalized to Gapdh mRNA levels. qRT-PCR primers were designed using Primer-BLAST to span across splice junctions, resulting in PCR amplicons that span at least one intron. Primer sequences are listed in Table S4.

To assess the fraction of candidate transcripts carrying A-to-I edits, RT-PCR was performed on Th17 cDNAs using the Q5 Hot Start High-Fidelity 2X Master Mix (New England Biolabs) with the standard thermocycling protocol. Samples were incubated at $98{ }^{\circ} \mathrm{C}$ for 30 seconds, followed by a denaturation for $10 \mathrm{~s}$ at $98^{\circ} \mathrm{C}, 20 \mathrm{~s}$ at $66{ }^{\circ} \mathrm{C}$, and 72 ${ }^{\circ} \mathrm{C}$ for $1 \mathrm{~min}$ for a total of 34 cycles. PCR products were purified using Quick PCR Purification Kit (Qiagen) and sequenced. Primers are listed in Table S4.

\section{Statistical Analysis}

All values are presented as means \pm SD. Significant differences were evaluated using GraphPad Prism 8 software. The Student's t-test or paired t-test were used to determine 
significant differences between two groups. A two-tailed p-value of $<0.05$ was considered statistically significant in all experiments.

\section{SUPPLEMENTAL INFORMATION}

Figure S1. Expression of genes encoding ADARs in different T helper subsets

A. RNA expression of Adar, Adarb1, and Adarb2 in naive and different polarized T helper subsets (Stubbington et al., 2015).

Figure S2. ADAR2 nuclear localization in Th17 cells

A. Representative western blot analysis of cytoplasmic and nuclear extracts from WT Th17 cells. This experiment was repeated two times on independent biological samples with similar results.

Figure S3. Chromatin accessibility of putative Adarb1 enhancers correlates with promoter openness

A. Normalized ATAC-seq read count at the Adarb1 promoter and putative cisregulatory elements in the 0 (naive), 48, or 72-hour time points were used to calculate co-openness correlation scores.

Figure S4. ADAR2 associates with Hif1a transcripts in Th17 cells

A. Wildtype Th17 cells polarized in culture for 72 hours were lysed and immunoprecipitated with control or ADAR2 specific antibodies. Hif1a pre-mRNA or mature mRNA in input and immunoprecipitated samples were quantified by qRT- 
PCR. Average and standard deviation from two biological replicates are shown. * $p$-value $<0.05$.

Figure S5. Splicing assessment of the Hif1a and Adarb1 transcripts

A. Primers designed to amplify Hif1a transcript (exon 11-intro 11 and exon 11-exon 12 junction) and Adrb1 transcript (exon 4-intron 4 and exon 4-exon 5 junction) by RT-PCR. Splicing frequency was calculated as the ratio of unspliced RNA over spliced mRNA in each condition.

Figure S6. Abundant RNA and protein expression of DDX5 in Th17 cells

A. RNA expression of transcripts encoding various RBPs in $\mathrm{CD}^{+}$naive and different polarized T helper subsets (Stubbington et al., 2015).

B. Western blot analysis confirmed efficient immunoprecipitation of DDX5 from two independent replicates of Th17 cell lysates.

Figure S7. Expression of select DDX5 dependent and independent genes in Th17 cells

A. DEseq2 quantified RNA expression of Batf, Irf4, and Rorc in Th17 cells from WT ${ }^{\top}$ $(n=2)$ and $\operatorname{DDX}^{\Delta \top}(n=2)$ mice. Each dot represents result from one biological replicate.

B. DEseq2 quantified RNA expression of Adar and Adarb1 in Th17 cells from WT ${ }^{\top}$ $(n=2)$ and $\operatorname{DDX}^{\Delta \top}(n=2)$ mice. Each dot represents result from one biological replicate. ${ }^{*} p$-value $<0.05,{ }^{* * * *} p$-value $<0.001$. 
Figure S8. Identification and validation of DDX5-dependent A-to-I editing in Th17 cells

A. Scatterplot of SAILOR analysis on two independent biological replicates of Th17 cells from $\mathrm{WT}^{\top}$ and $\mathrm{DDX} 5^{\Delta \top}$ mice.

B. Results from SAILOR analysis on Hif1a and Apobec3 transcripts in two pairs of $\mathrm{WT}^{\top}$ and DDX5 ${ }^{\Delta \mathrm{T}} \mathrm{Th} 17$ cells.

C. Representative RT-PCR and Sanger sequencing analyses of the Apobec3 RNAs from two additional pairs of $\mathrm{WT}^{\top}$ and $\mathrm{DDX} 5^{\Delta \mathrm{T}}$ Th17 samples independent from B.

Table S1. SAILOR analysis of CD4 ${ }^{+}$naive and Th17 cells

Table S2. SAILOR analysis of Th17 cells from $\mathrm{WT}^{\top}$ and $\mathrm{DDX} 5^{\Delta \mathrm{T}}$ mice

Table S3. sgRNA information

Table S4. Primer information

Table S5. Antibody information 


\section{REFERENCES}

Anantharaman, A., Tripathi, V., Khan, A., Yoon, J.H., Singh, D.K., Gholamalamdari, O.,

Guang, S., Ohlson, J., Wahlstedt, H., Ohman, M., et al. (2017). ADAR2 regulates RNA stability by modifying access of decay-promoting RNA-binding proteins. Nucleic Acids Res 45, 4189-4201.

Aschenbrenner, D., Foglierini, M., Jarrossay, D., Hu, D., Weiner, H.L., Kuchroo, V.K., Lanzavecchia, A., Notarbartolo, S., and Sallusto, F. (2018). An immunoregulatory and tissue-residency program modulated by c-MAF in human TH17 cells. Nat Immunol 19, $1126-1136$.

Athanasiadis, A., Rich, A., and Maas, S. (2004). Widespread A-to-I RNA editing of Alucontaining mRNAs in the human transcriptome. PLoS Biol 2, e391.

Bellemore, S.M., Nikoopour, E., Schwartz, J.A., Krougly, O., Lee-Chan, E., and Singh, B. (2015). Preventative role of interleukin-17 producing regulatory T helper type 17 (Treg 17) cells in type 1 diabetes in non-obese diabetic mice. Clin Exp Immunol 182, 261-269.

Buenrostro, J.D., Wu, B., Chang, H.Y., and Greenleaf, W.J. (2015). ATAC-seq: A Method for Assaying Chromatin Accessibility Genome-Wide. Curr Protoc Mol Biol 109, 212921 212929.

Chalk, A.M., Taylor, S., Heraud-Farlow, J.E., and Walkley, C.R. (2019). The majority of A-to-I RNA editing is not required for mammalian homeostasis. Genome Biol 20, 268.

Chaudhry, A., Samstein, R.M., Treuting, P., Liang, Y., Pils, M.C., Heinrich, J.M., Jack, R.S., Wunderlich, F.T., Bruning, J.C., Muller, W., et al. (2011). Interleukin-10 signaling in regulatory $\mathrm{T}$ cells is required for suppression of Th17 cell-mediated inflammation. Immunity $34,566-578$. 
Ciofani, M., Madar, A., Galan, C., Sellars, M., Mace, K., Pauli, F., Agarwal, A., Huang, W., Parkhurst, C.N., Muratet, M., et al. (2012). A validated regulatory network for Th17 cell specification. Cell 151, 289-303.

Codarri, L., Gyulveszi, G., Tosevski, V., Hesske, L., Fontana, A., Magnenat, L., Suter, T., and Becher, B. (2011). RORgammat drives production of the cytokine GM-CSF in helper T cells, which is essential for the effector phase of autoimmune neuroinflammation. Nat Immunol 12, 560-567.

Dang, E.V., Barbi, J., Yang, H.Y., Jinasena, D., Yu, H., Zheng, Y., Bordman, Z., Fu, J., Kim, Y., Yen, H.R., et al. (2011). Control of T(H)17/T(reg) balance by hypoxia-inducible factor 1. Cell 146, 772-784.

Dobin, A., Davis, C.A., Schlesinger, F., Drenkow, J., Zaleski, C., Jha, S., Batut, P., Chaisson, M., and Gingeras, T.R. (2013). STAR: ultrafast universal RNA-seq aligner. Bioinformatics 29, 15-21.

Esplugues, E., Huber, S., Gagliani, N., Hauser, A.E., Town, T., Wan, Y.Y., O'Connor, W., Jr., Rongvaux, A., Van Rooijen, N., Haberman, A.M., et al. (2011). Control of TH17 cells occurs in the small intestine. Nature $475,514-518$.

Gallo, A., Vukic, D., Michalik, D., O'Connell, M.A., and Keegan, L.P. (2017). ADAR RNA editing in human disease; more to it than meets the I. Hum Genet 136, 1265-1278.

Higuchi, M., Maas, S., Single, F.N., Hartner, J., Rozov, A., Burnashev, N., Feldmeyer, D., Sprengel, R., and Seeburg, P.H. (2000). Point mutation in an AMPA receptor gene rescues lethality in mice deficient in the RNA-editing enzyme ADAR2. Nature 406, 78-81. Hueber, W., Sands, B.E., Lewitzky, S., Vandemeulebroecke, M., Reinisch, W., Higgins, P.D., Wehkamp, J., Feagan, B.G., Yao, M.D., Karczewski, M., et al. (2012). Secukinumab, 
a human anti-IL-17A monoclonal antibody, for moderate to severe Crohn's disease: unexpected results of a randomised, double-blind placebo-controlled trial. Gut 61, 16931700.

Kaikkonen, M.U., Spann, N.J., Heinz, S., Romanoski, C.E., Allison, K.A., Stender, J.D., Chun, H.B., Tough, D.F., Prinjha, R.K., Benner, C., et al. (2013). Remodeling of the enhancer landscape during macrophage activation is coupled to enhancer transcription. Mol Cell 51, 310-325.

Labun, K., Montague, T.G., Krause, M., Torres Cleuren, Y.N., Tjeldnes, H., and Valen, E. (2019). CHOPCHOP v3: expanding the CRISPR web toolbox beyond genome editing. Nucleic Acids Res 47, W171-W174.

Landt, S.G., Marinov, G.K., Kundaje, A., Kheradpour, P., Pauli, F., Batzoglou, S., Bernstein, B.E., Bickel, P., Brown, J.B., Cayting, P., et al. (2012). ChIP-seq guidelines and practices of the ENCODE and modENCODE consortia. Genome Res 22, 1813-1831. Li, H., Handsaker, B., Wysoker, A., Fennell, T., Ruan, J., Homer, N., Marth, G., Abecasis, G., Durbin, R., and Genome Project Data Processing, S. (2009). The Sequence Alignment/Map format and SAMtools. Bioinformatics 25, 2078-2079.

Liao, Y., Smyth, G.K., and Shi, W. (2014). featureCounts: an efficient general purpose program for assigning sequence reads to genomic features. Bioinformatics 30, 923-930. Lin, C., Yang, L., Yang, J.J., Huang, Y., and Liu, Z.R. (2005). ATPase/helicase activities of p68 RNA helicase are required for pre-mRNA splicing but not for assembly of the spliceosome. Mol Cell Biol 25, 7484-7493.

Liu, Z.R. (2002). p68 RNA helicase is an essential human splicing factor that acts at the U1 snRNA-5' splice site duplex. Mol Cell Biol 22, 5443-5450. 
Love, M.I., Huber, W., and Anders, S. (2014). Moderated estimation of fold change and dispersion for RNA-seq data with DESeq2. Genome Biol 15, 550.

Loven, J., Hoke, H.A., Lin, C.Y., Lau, A., Orlando, D.A., Vakoc, C.R., Bradner, J.E., Lee, T.I., and Young, R.A. (2013). Selective inhibition of tumor oncogenes by disruption of super-enhancers. Cell 153, 320-334.

McGeachy, M.J., Bak-Jensen, K.S., Chen, Y., Tato, C.M., Blumenschein, W., McClanahan, T., and Cua, D.J. (2007). TGF-beta and IL-6 drive the production of IL-17 and IL-10 by $\mathrm{T}$ cells and restrain $\mathrm{T}(\mathrm{H})-17$ cell-mediated pathology. Nat Immunol $8,1390-$ 1397.

Meng, X., Grotsch, B., Luo, Y., Knaup, K.X., Wiesener, M.S., Chen, X.X., Jantsch, J., Fillatreau, S., Schett, G., and Bozec, A. (2018). Hypoxia-inducible factor-1alpha is a critical transcription factor for IL-10-producing B cells in autoimmune disease. Nat Commun 9, 251.

Morita, S., Kojima, T., and Kitamura, T. (2000). Plat-E: an efficient and stable system for transient packaging of retroviruses. Gene therapy 7, 1063-1066.

Nakahama, T., Kato, Y., Kim, J.I., Vongpipatana, T., Suzuki, Y., Walkley, C.R., and Kawahara, Y. (2018). ADAR1-mediated RNA editing is required for thymic self-tolerance and inhibition of autoimmunity. EMBO Rep 19.

Nicol, S.M., Bray, S.E., Black, H.D., Lorimore, S.A., Wright, E.G., Lane, D.P., Meek, D.W., Coates, P.J., and Fuller-Pace, F.V. (2013). The RNA helicase p68 (DDX5) is selectively required for the induction of p53-dependent p21 expression and cell-cycle arrest after DNA damage. Oncogene 32, 3461-3469. 
Nicol, S.M., Bray, S.E., Derek Black, H., Lorimore, S.A., Wright, E.G., Lane, D.P., Meek, D.W., Coates, P.J., and Fuller-Pace, F.V. (2012). The RNA helicase p68 (DDX5) is selectively required for the induction of p53-dependent p21 expression and cell-cycle arrest after DNA damage. Oncogene.

Palladino, M.J., Keegan, L.P., O'Connell, M.A., and Reenan, R.A. (2000). dADAR, a Drosophila double-stranded RNA-specific adenosine deaminase is highly developmentally regulated and is itself a target for RNA editing. RNA 6, 1004-1018.

Qi, L., Song, Y., Chan, T.H.M., Yang, H., Lin, C.H., Tay, D.J.T., Hong, H., Tang, S.J., Tan, K.T., Huang, X.X., et al. (2017). An RNA editing/dsRNA binding-independent gene regulatory mechanism of ADARs and its clinical implication in cancer. Nucleic Acids Res 45, 10436-10451.

Qiu, S., Li, W., Xiong, H., Liu, D., Bai, Y., Wu, K., Zhang, X., Yang, H., Ma, K., Hou, Y., et al. (2016). Single-cell RNA sequencing reveals dynamic changes in A-to-I RNA editome during early human embryogenesis. BMC Genomics 17, 766.

Ran, F.A., Hsu, P.D., Wright, J., Agarwala, V., Scott, D.A., and Zhang, F. (2013). Genome engineering using the CRISPR-Cas9 system. Nat Protoc 8, 2281-2308.

Reiner, S.L. (2009). Decision making during the conception and career of CD4+ T cells. Nat Rev Immunol 9, 81-82.

Stockinger, B., and Omenetti, S. (2017). The dichotomous nature of T helper 17 cells. Nat Rev Immunol 17, 535-544.

Stubbington, M.J., Mahata, B., Svensson, V., Deonarine, A., Nissen, J.K., Betz, A.G., and Teichmann, S.A. (2015). An atlas of mouse CD4(+) T cell transcriptomes. Biol Direct 10, 14. 
Tan, M.H., Li, Q., Shanmugam, R., Piskol, R., Kohler, J., Young, A.N., Liu, K.I., Zhang, R., Ramaswami, G., Ariyoshi, K., et al. (2017). Dynamic landscape and regulation of RNA editing in mammals. Nature 550, 249-254.

Tanaka, S., Jiang, Y., Martinez, G.J., Tanaka, K., Yan, X., Kurosaki, T., Kaartinen, V., Feng, X.H., Tian, Q., Wang, X., et al. (2018). Trim33 mediates the proinflammatory function of Th17 cells. J Exp Med 215, 1853-1868.

Tang, S.J., Shen, H., An, O., Hong, H., Li, J., Song, Y., Han, J., Tay, D.J.T., Ng, V.H.E., Bellido Molias, F., et al. (2020). Cis- and trans-regulations of pre-mRNA splicing by RNA editing enzymes influence cancer development. Nat Commun 11, 799.

Thorvaldsdottir, H., Robinson, J.T., and Mesirov, J.P. (2013). Integrative Genomics Viewer (IGV): high-performance genomics data visualization and exploration. Brief Bioinform 14, 178-192.

Tigno-Aranjuez, J.T., Jaini, R., Tuohy, V.K., Lehmann, P.V., and Tary-Lehmann, M. (2009). Encephalitogenicity of complete Freund's adjuvant relative to CpG is linked to induction of Th17 cells. J Immunol 183, 5654-5661.

Van Nostrand, E.L., Pratt, G.A., Shishkin, A.A., Gelboin-Burkhart, C., Fang, M.Y., Sundararaman, B., Blue, S.M., Nguyen, T.B., Surka, C., Elkins, K., et al. (2016). Robust transcriptome-wide discovery of RNA-binding protein binding sites with enhanced CLIP (eCLIP). Nat Methods 13, 508-514.

Volchenkov, R., Nygaard, V., Sener, Z., and Skalhegg, B.S. (2017). Th17 Polarization under Hypoxia Results in Increased IL-10 Production in a Pathogen-Independent Manner. Front Immunol 8, 698. 
Walkley, C.R., and Li, J.B. (2017). Rewriting the transcriptome: adenosine-to-inosine RNA editing by ADARs. Genome Biol 18, 205.

Washburn, M.C., Kakaradov, B., Sundararaman, B., Wheeler, E., Hoon, S., Yeo, G.W., and Hundley, H.A. (2014). The dsRBP and inactive editor ADR-1 utilizes dsRNA binding to regulate A-to-I RNA editing across the C. elegans transcriptome. Cell Rep 6, 599-607. Wu, X., Tian, J., and Wang, S. (2018). Insight Into Non-Pathogenic Th17 Cells in Autoimmune Diseases. Front Immunol 9, 1112.

Yasuda, K., Takeuchi, Y., and Hirota, K. (2019). The pathogenicity of Th17 cells in autoimmune diseases. Semin Immunopathol 41, 283-297.

Zhang, Y., Liu, T., Meyer, C.A., Eeckhoute, J., Johnson, D.S., Bernstein, B.E., Nusbaum, C., Myers, R.M., Brown, M., Li, W., et al. (2008). Model-based analysis of ChIP-Seq (MACS). Genome Biol 9, R137.

Zhou, B., Li, X., Luo, D., Lim, D.H., Zhou, Y., and Fu, X.D. (2019). GRID-seq for comprehensive analysis of global RNA-chromatin interactions. Nat Protoc 14, 2036-2068. 
Figure 1. An intragenic super enhancer promotes Adarb1 transcription in Th17 cells

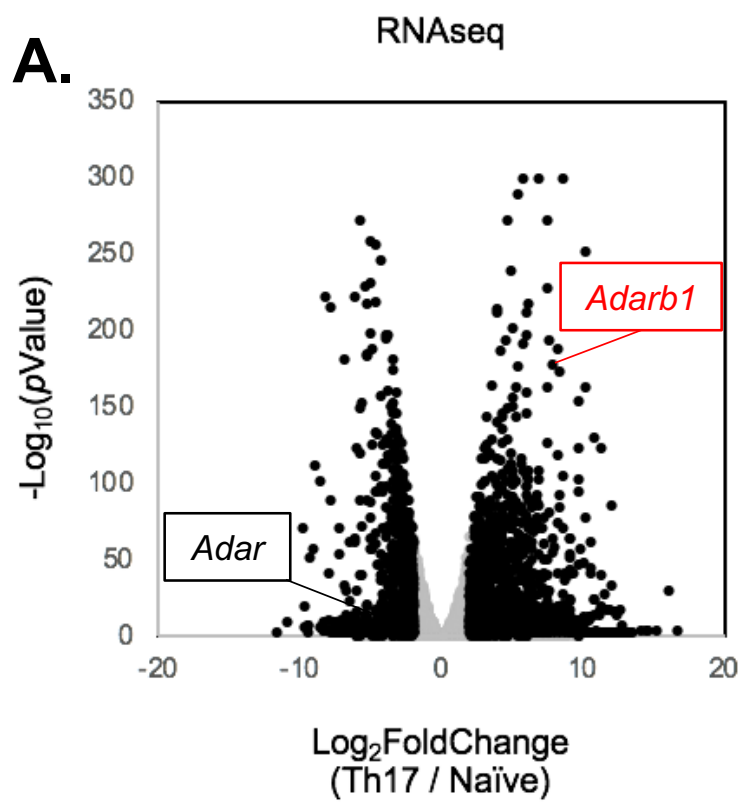

D.

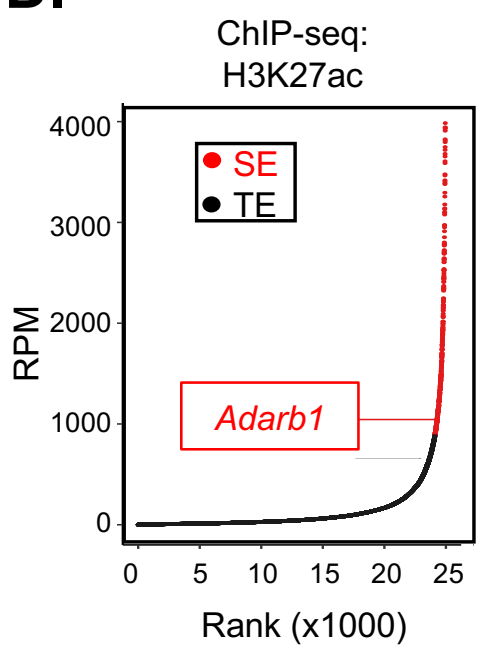

$\mathbf{F}$

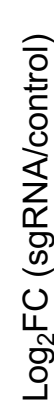

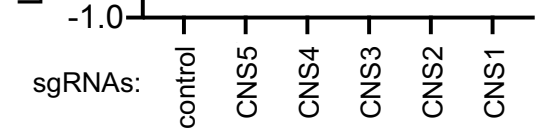

0

Adarb1 G.
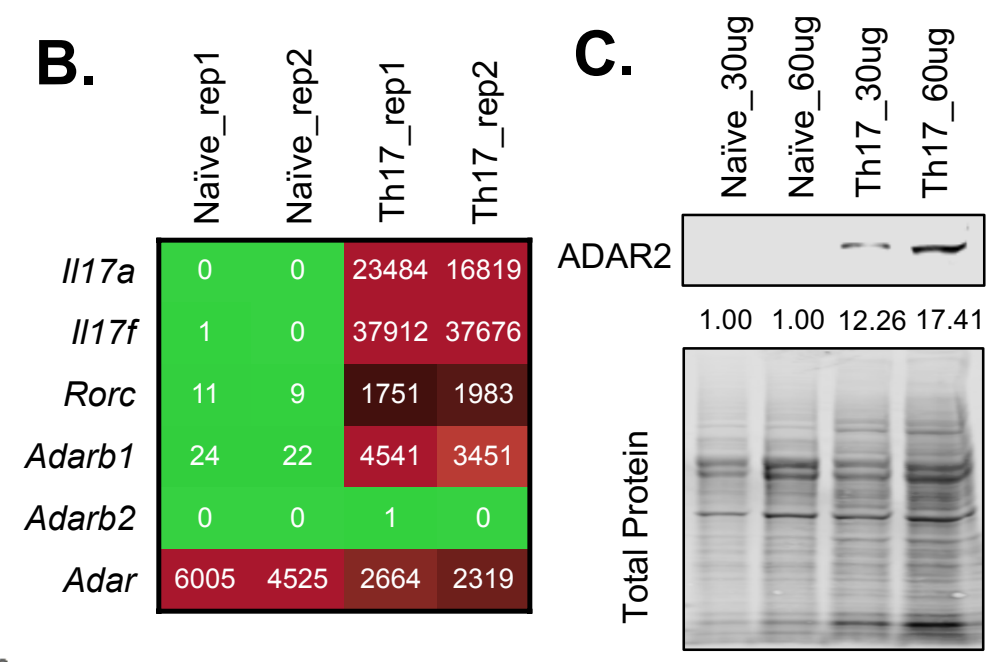

E. ChIP-seq: Th17 72hr_rep1
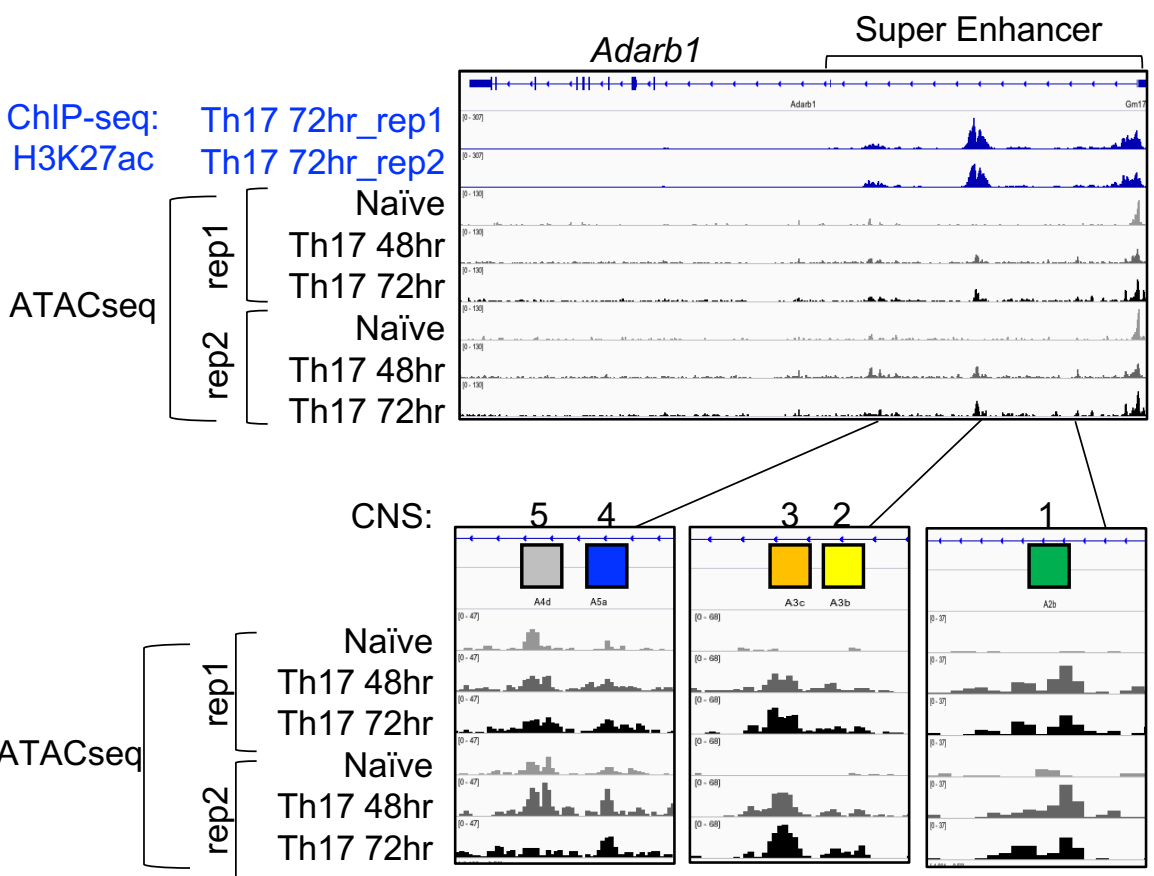

ATACseq

Adarb1

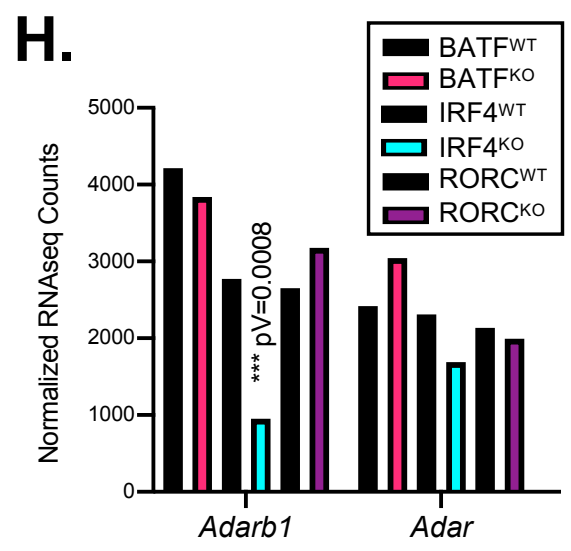


Figure 2. Overlapping and unique A-to-I editomes in wild type naive and Th17 cells

A.

Naïve

A-to-I edited sites (571)

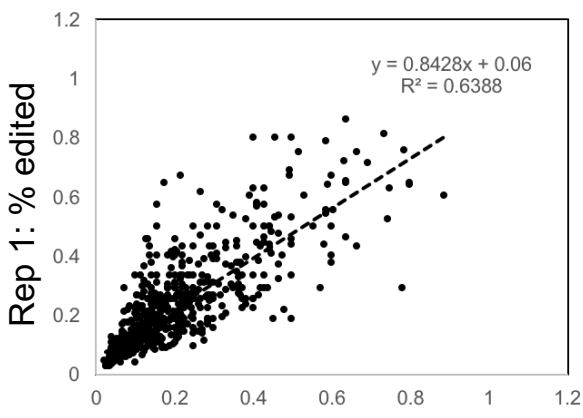

Rep 2: \% edited
Th17

A-to-I edited sites (399)

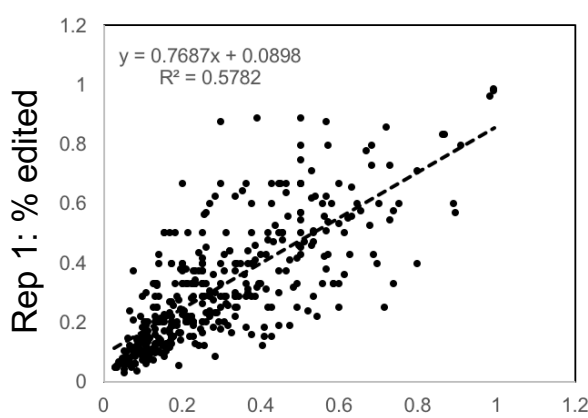

Rep 2: \% edited
B.

A-to-I edited sites

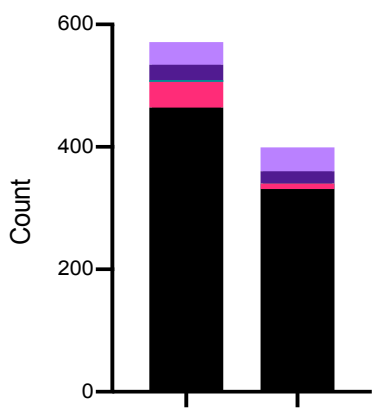

Naïve Th17

(571)

(399)

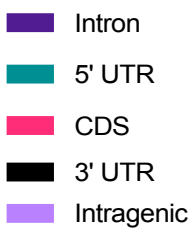

Naïve (270 transcripts)

Th17 (131 transcripts)

\section{口. Fold enrichment
$-\quad-\log 10 p$ Value}

protein autoubiquitination

response to steroid hormone stimulus

positive regulation of angiogenesis

T cell receptor signaling pathway activation of MAPK activity

$$
\text { - } \log _{10}(p \text { Value })
$$

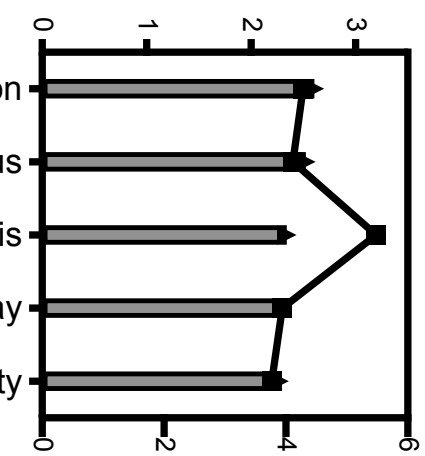

Fold Enrichment

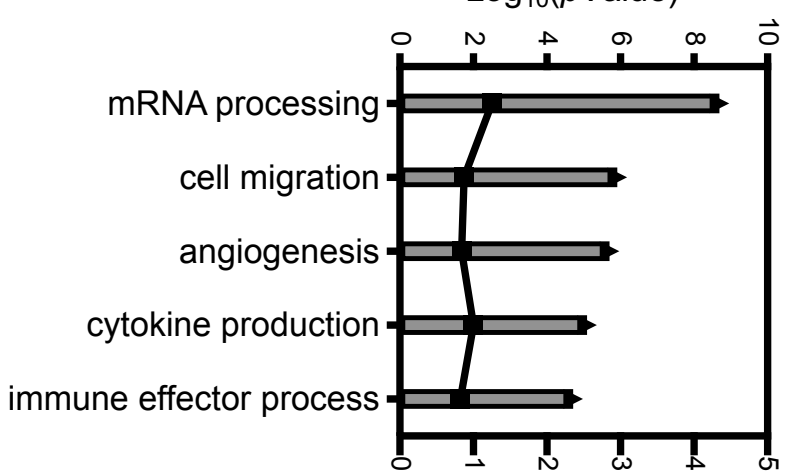

Fold Enrichment
D.

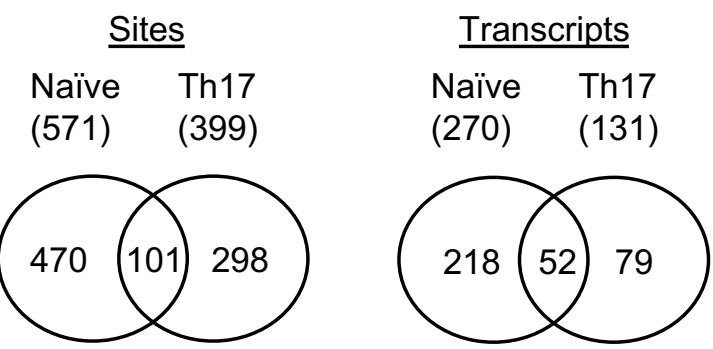

E.

DEseq

SAILOR

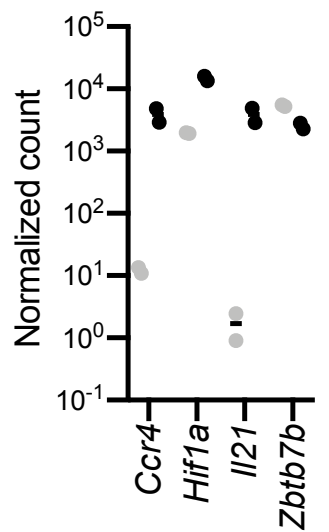


A. B. $\quad$ C.

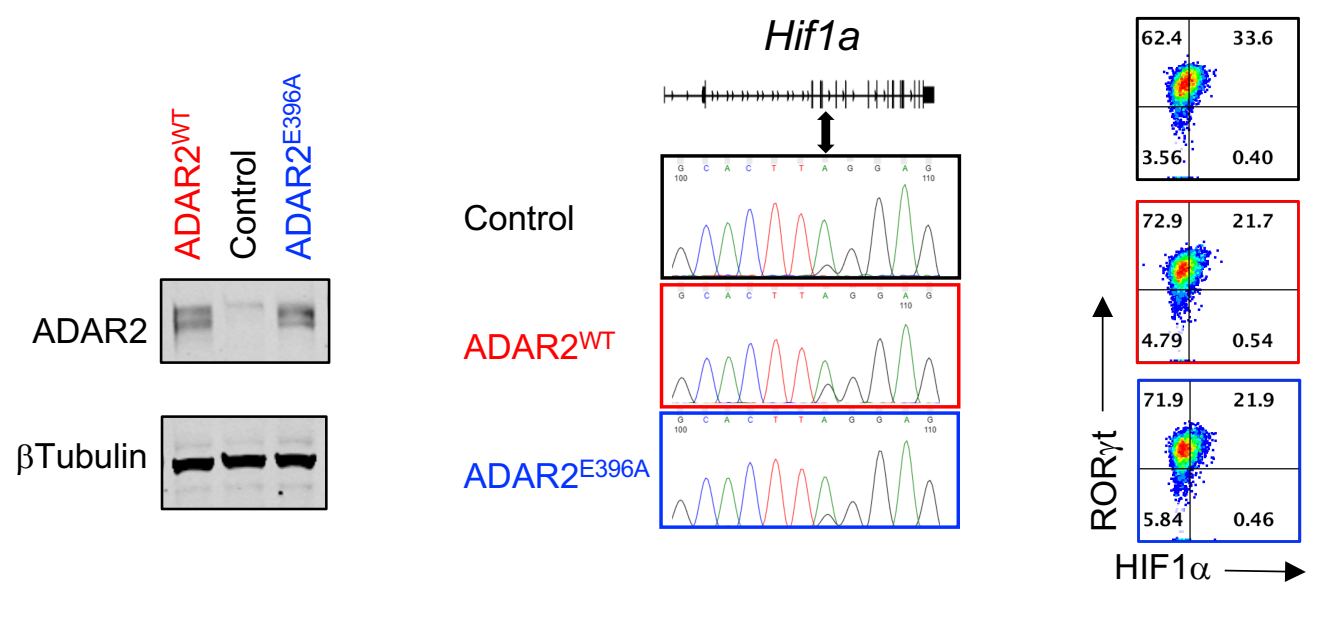

E

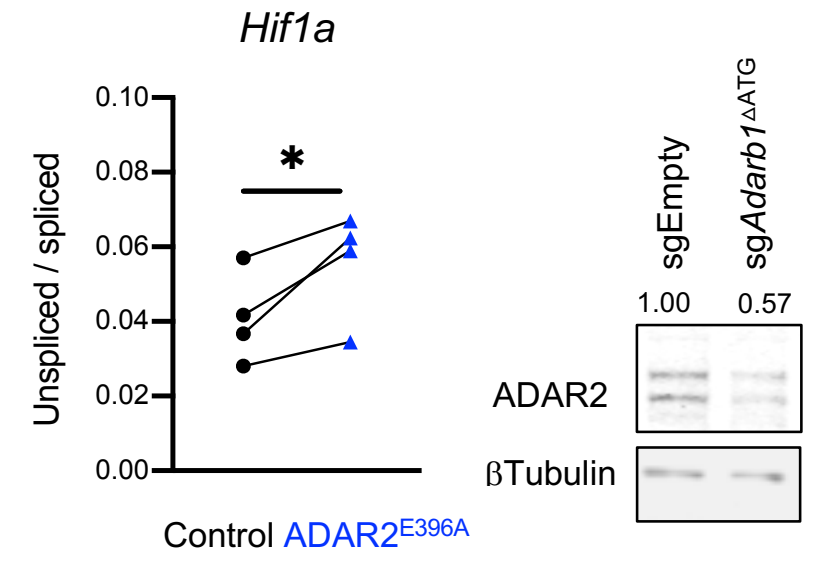

G.

F.

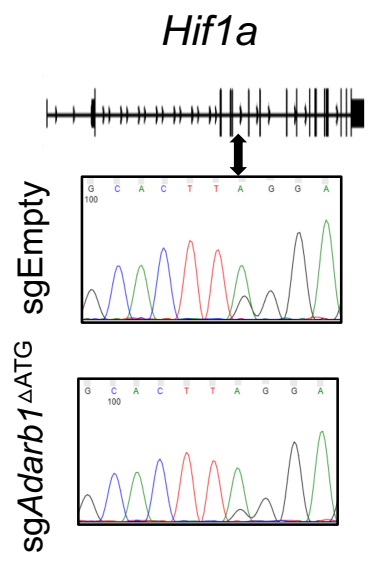

H.

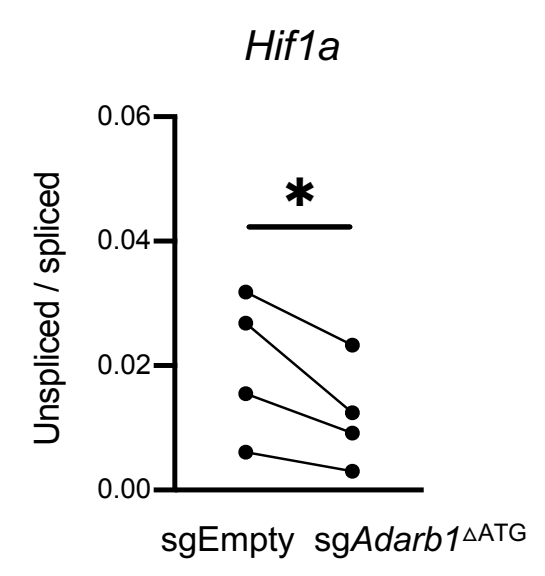


Figure 4. ADAR2-HIF1 $\alpha$ regulates IL-10 production in Th17 cells in vitro and in vivo
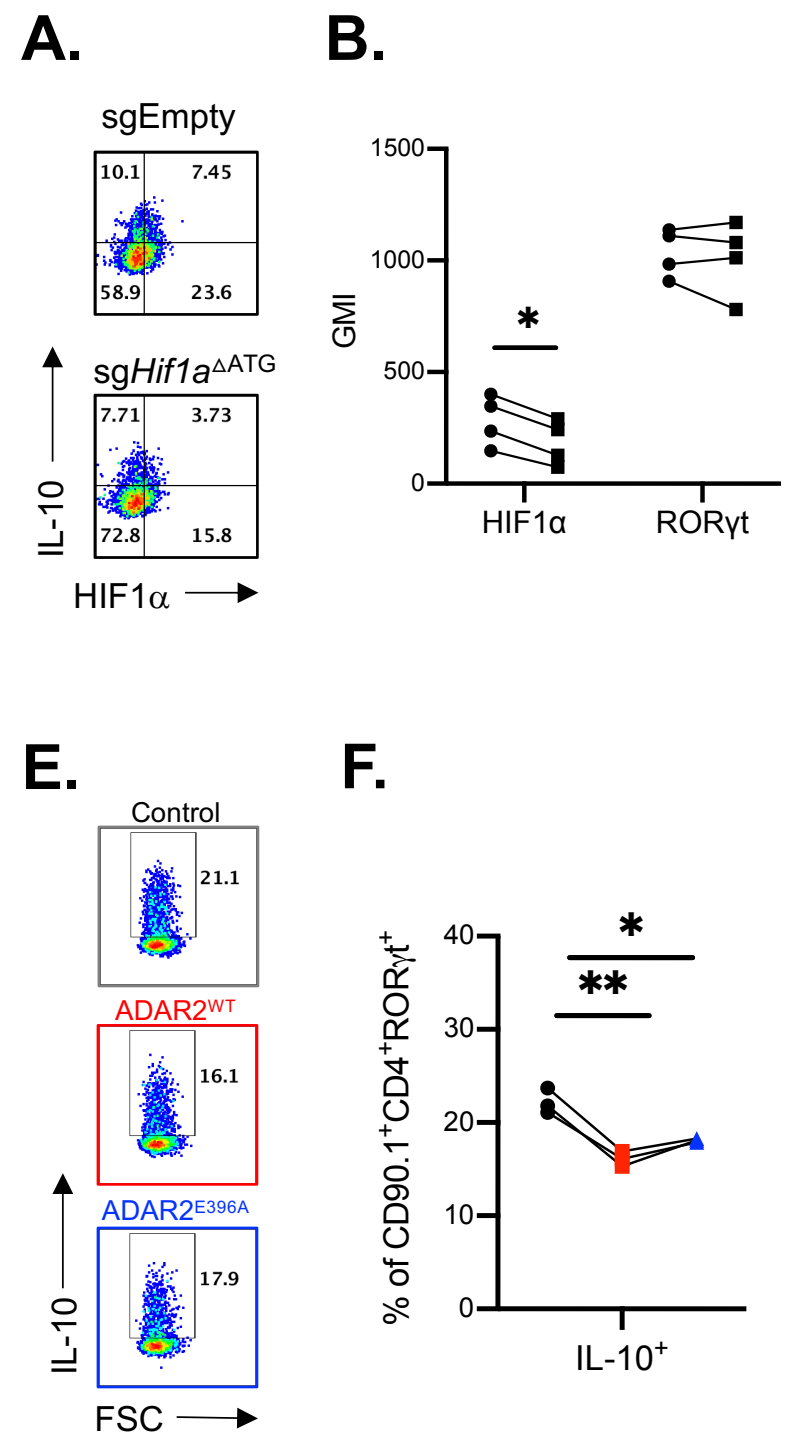

H.

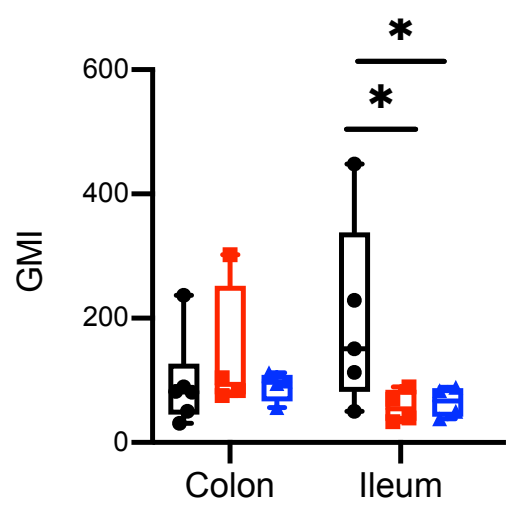

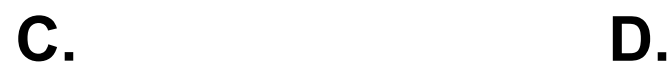
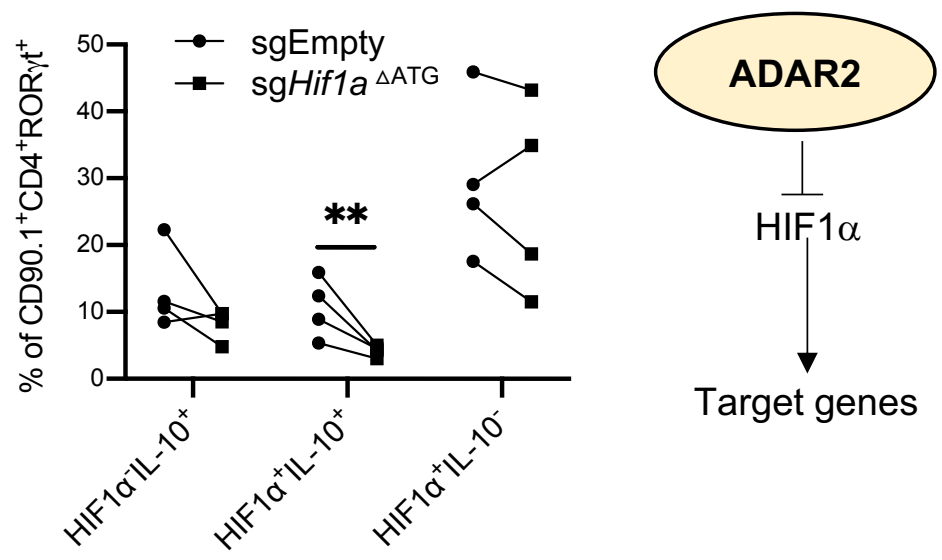

Target genes
F.

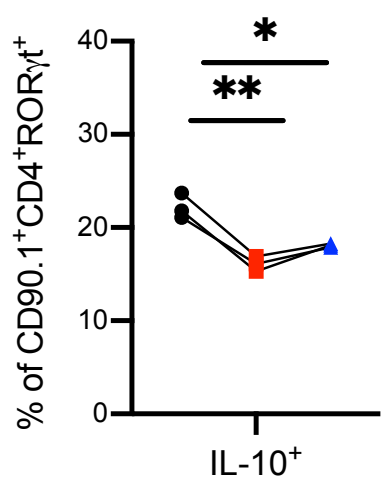

I.

$\operatorname{ROR} \gamma \mathrm{t}^{+} \mathrm{HIF} 1 \alpha^{+}$

J.

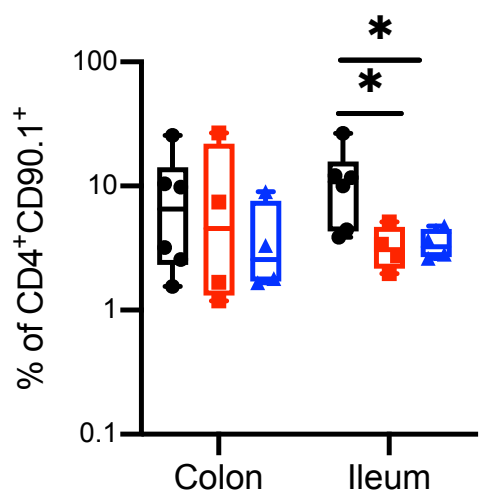

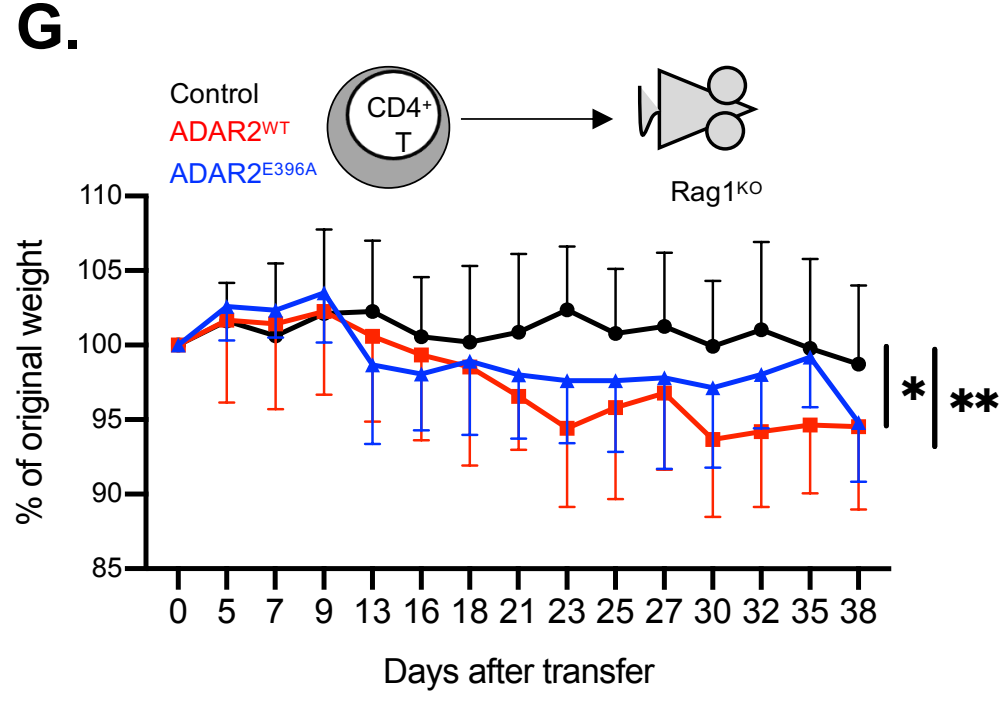
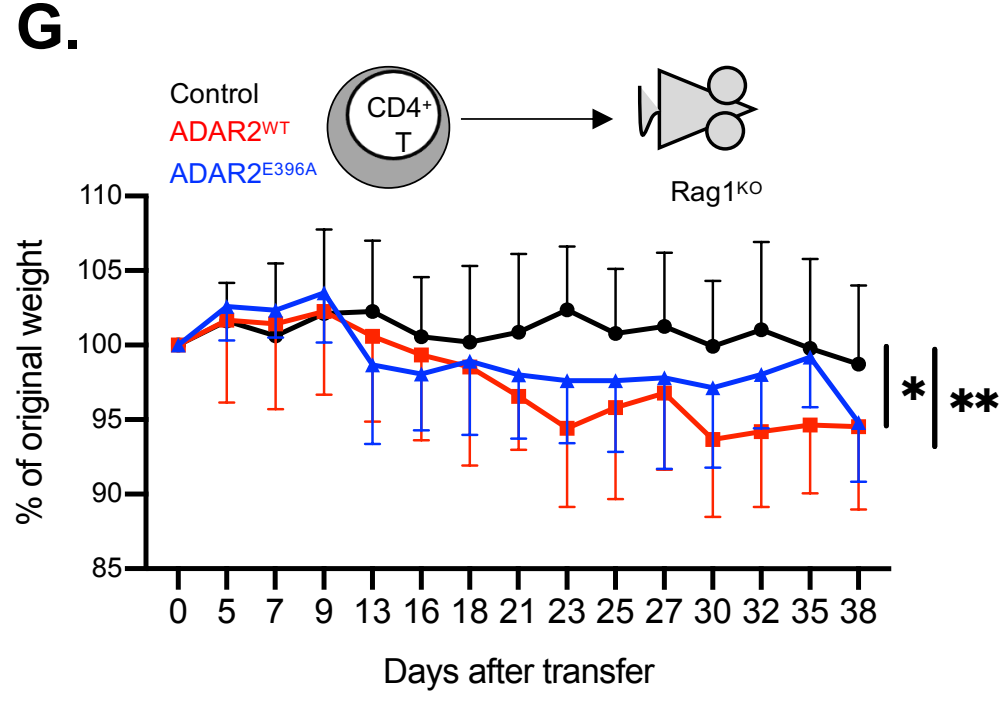

Days after transfer 
Figure 5. Adarb1 is post-transcriptionally regulated by RNA binding protein DDX5

A. B.
Anti-DDX5 \

RNA

$$
\text { UV } \rightarrow \text { Cells }
$$
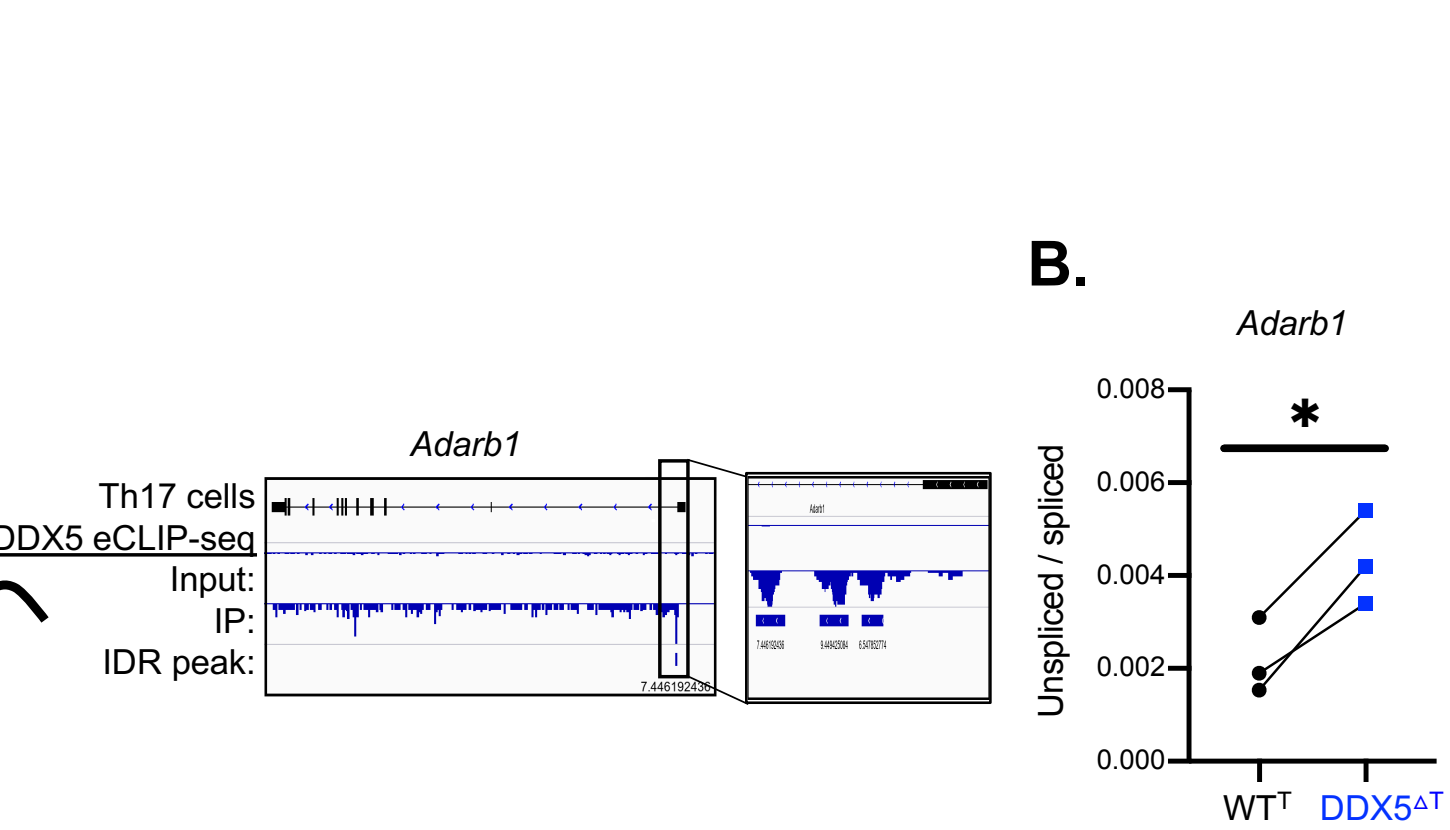

C.

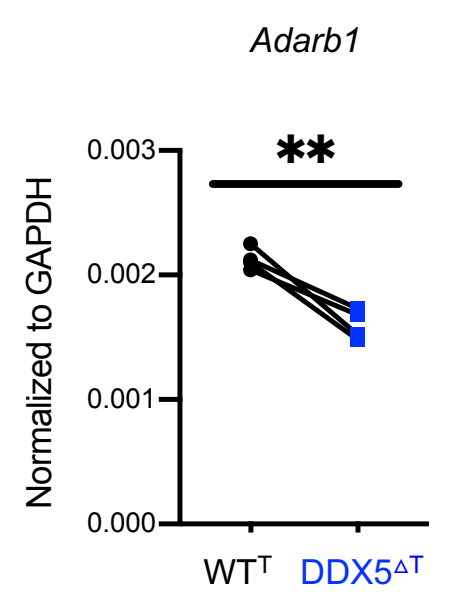

D.

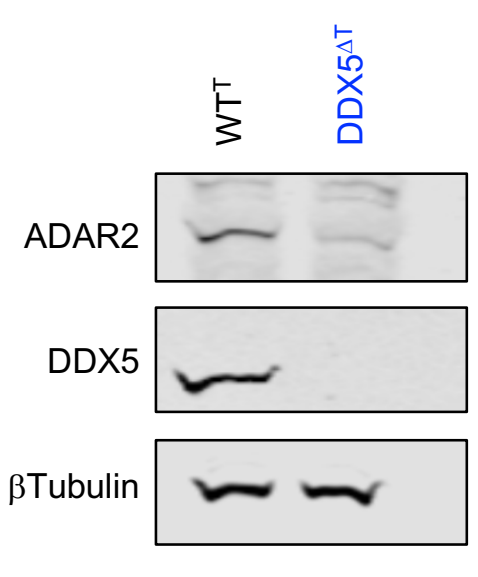

E.
RNAseq (SAILOR) A-I-editing $\log _{2}$ fold change: DDX5 ${ }^{\Delta T} / W^{\top}$

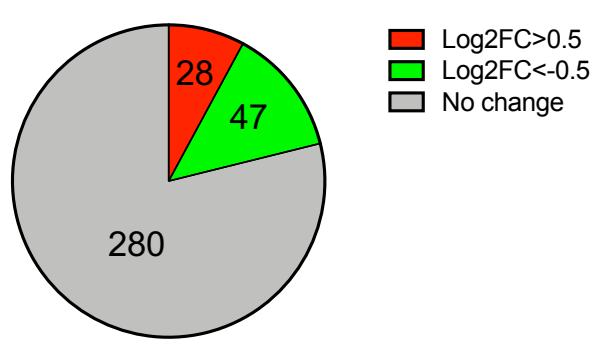

Total: 355 
Figure 6. DDX5 promotes HIF1a-IL-10 axis in vitro and in vivo

A. Hif1a
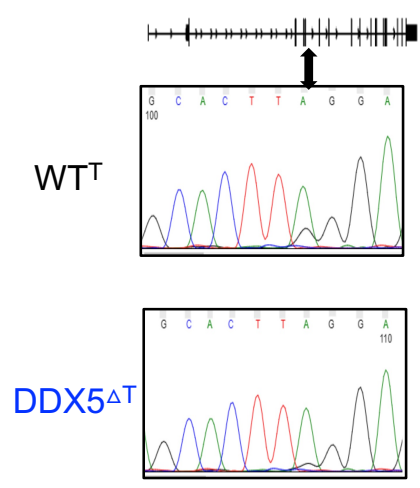

D.

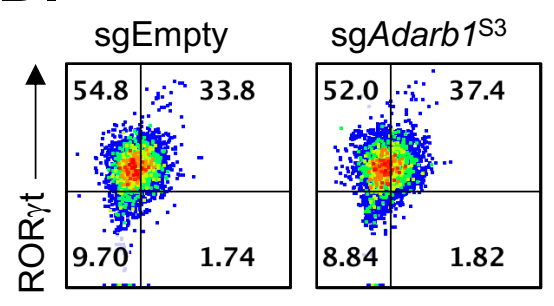
$\mathrm{HIF} 1 \alpha \longrightarrow$
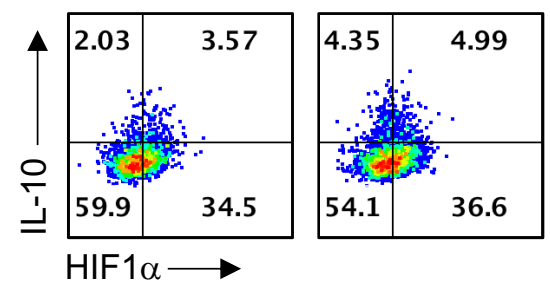

$\mathrm{HIF} 1 \alpha \longrightarrow$

$\mathbf{F}$

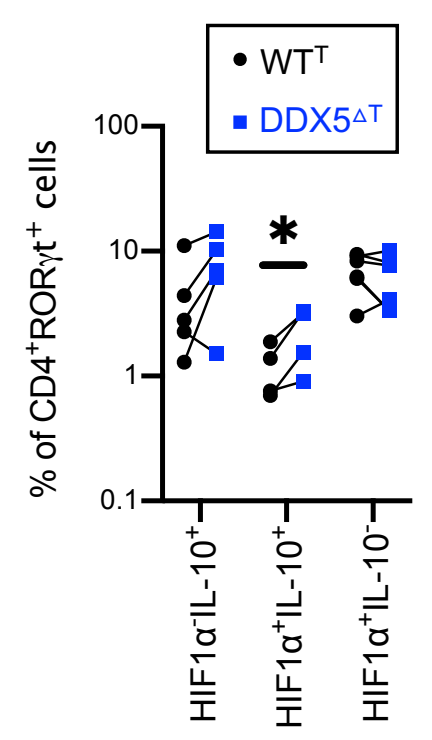

B.

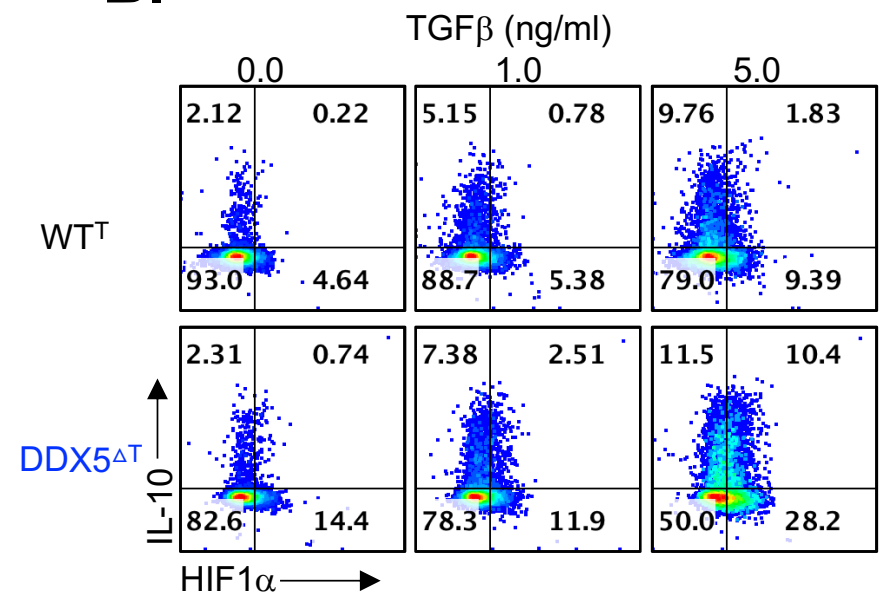

E.
C.

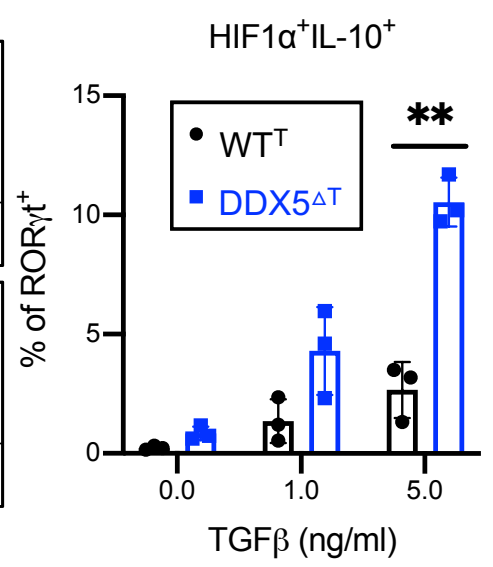

HIF1 $\alpha$

HIF $1 \alpha^{+} \mathrm{IL}-10^{+}$
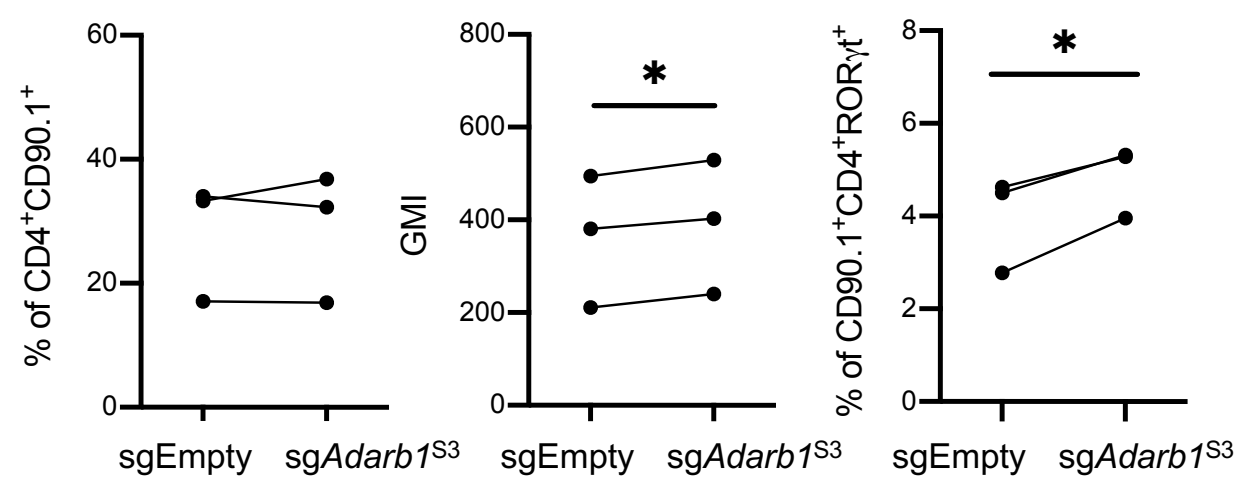

G.

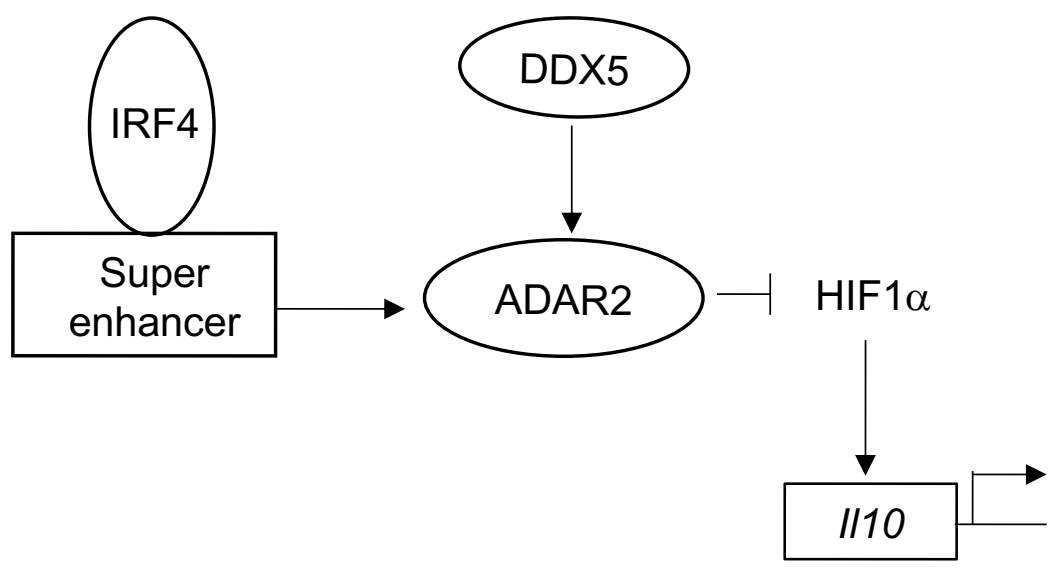


Supplementary Figures 
Figure S1. ADARs gene expression in different T helper subsets

A.

Th-express.org

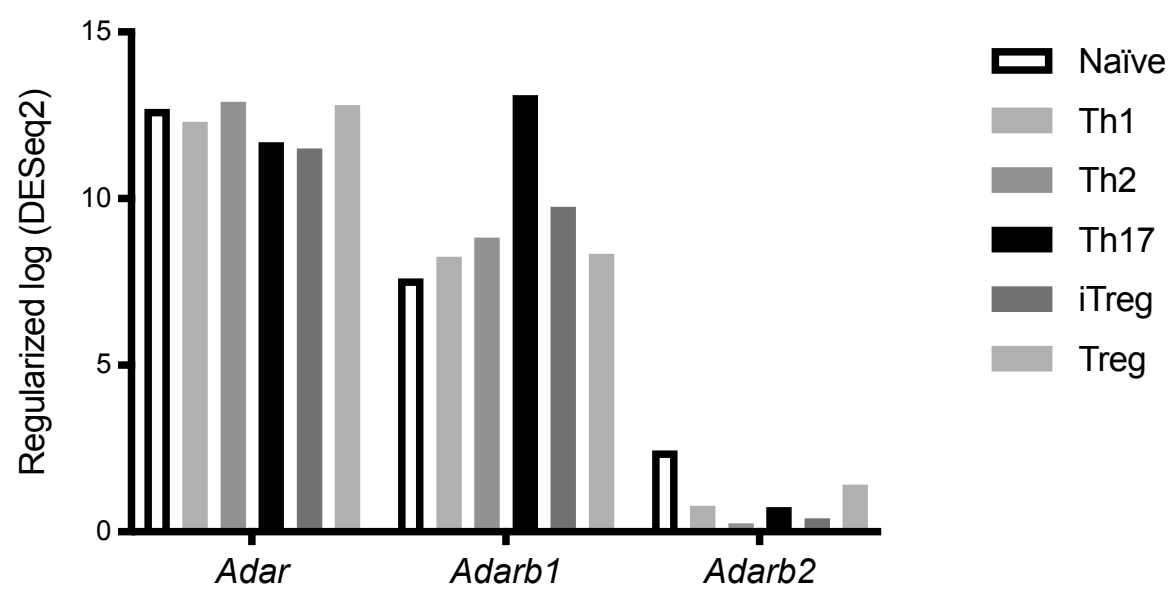


Figure S2. ADAR2 nuclear localization in Th17 cells

A.

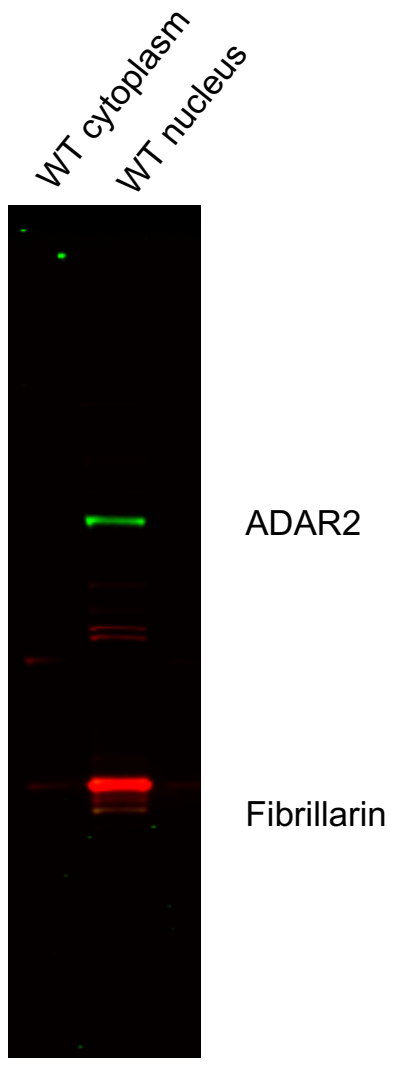

$\cdots$ TTubulin 
Figure S3. Chromatin accessibility of putative Adarb1 enhancers correlates with promoter openness

A.

Promoter-Enhancer Co-openness

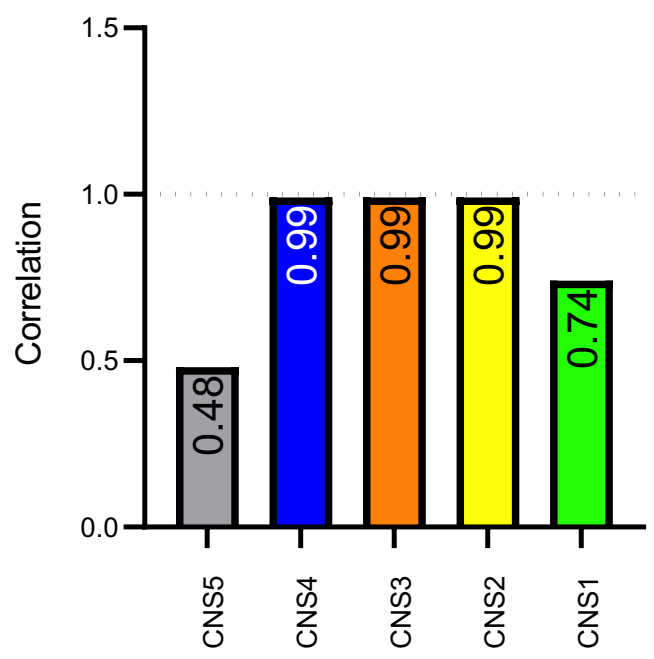


Figure S4. Hif1a transcript associates with ADAR2 in Th17 cells

A.

Hif1a pre-mRNA

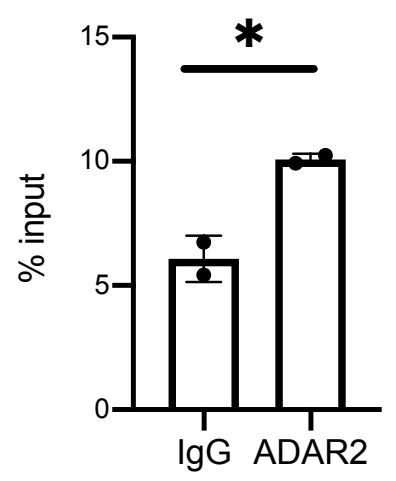

Hif1a mRNA

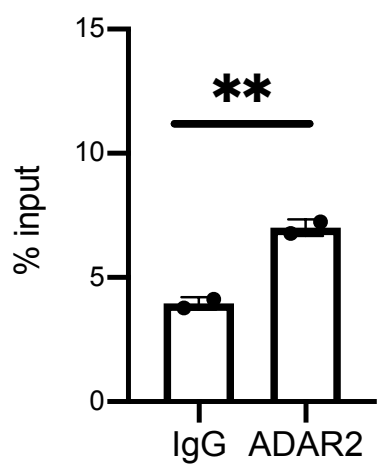


A.

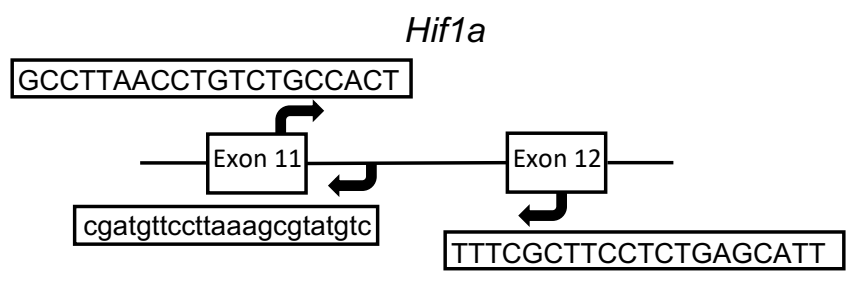

$\frac{\text { Hif } 1 \text { a unspliced } m R N A}{\text { Hif } 1 \text { a spliced } m R N A}=$ Hif1a splicing ratio

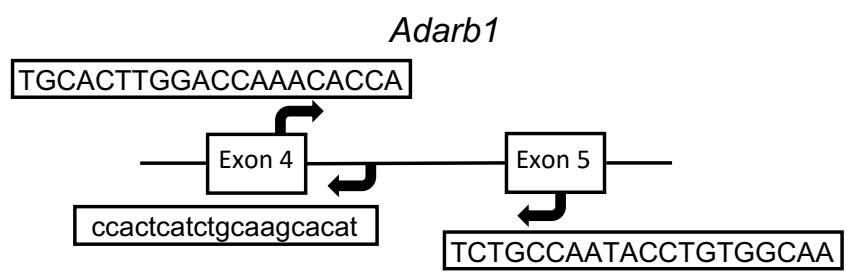

$\frac{\text { Adarb1 unspliced } m R N A}{\text { Adarb1 spliced } m R N A}=$ Adarb1 splicing ratio 
Figure S6. Abundant RNA and protein expression of DDX5 in Th17 cells

A.

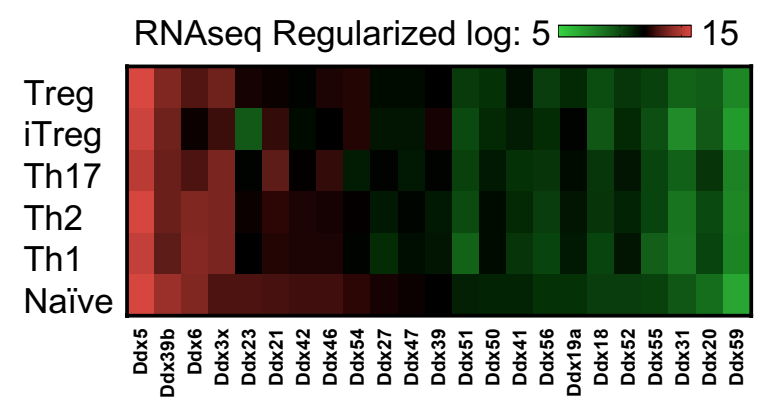

B.

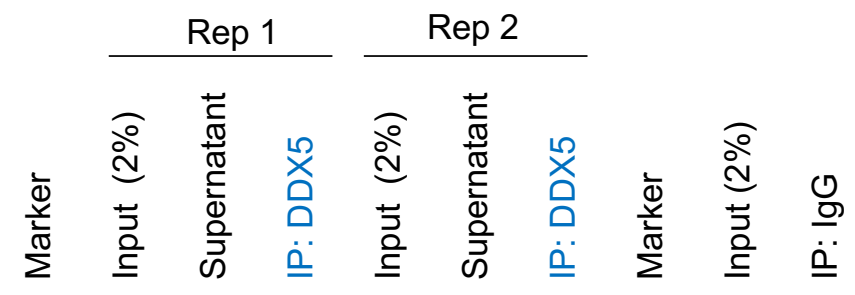

DDX5 (68kDa) > 
Figure S7. Expression of select DDX5 dependent and independent genes in Th17 cells

A.

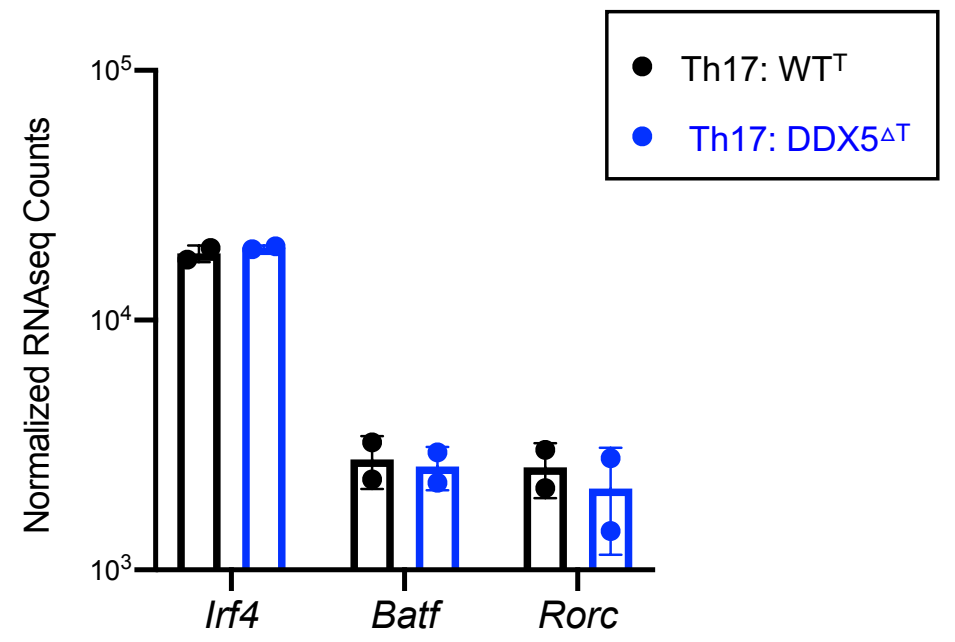

B.

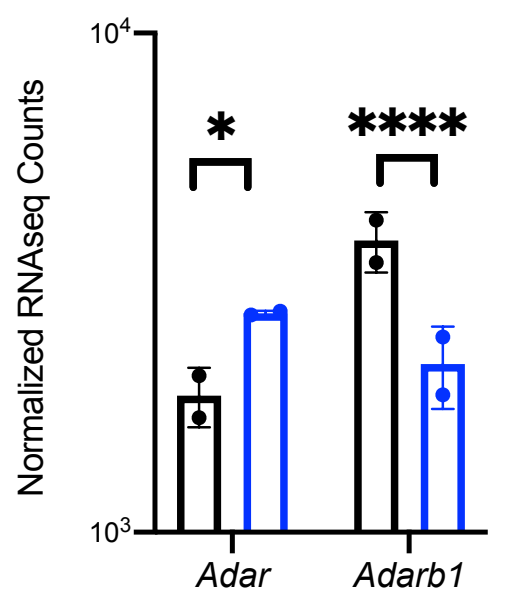



editing in Th17 cells

A.

B.

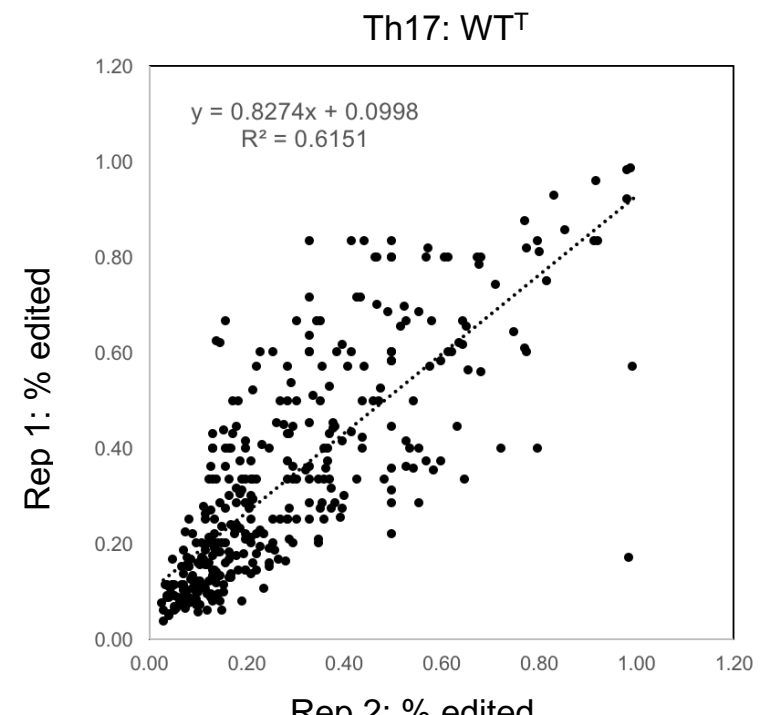

Rep 2: \% edited

\section{Hif1a}

\begin{tabular}{|c|c|}
\hline RNAseq & A-to-I Editing \\
\hline WT $^{\top}$ rep1 & 20.0 \\
\hline DDX5 $^{\Delta}$ ' rep1 & 7.0 \\
\hline WT $^{\top}$ rep2 & 29.8 \\
\hline DDX5 ${ }^{\Delta}$ rep2 & 2.6 \\
\hline
\end{tabular}

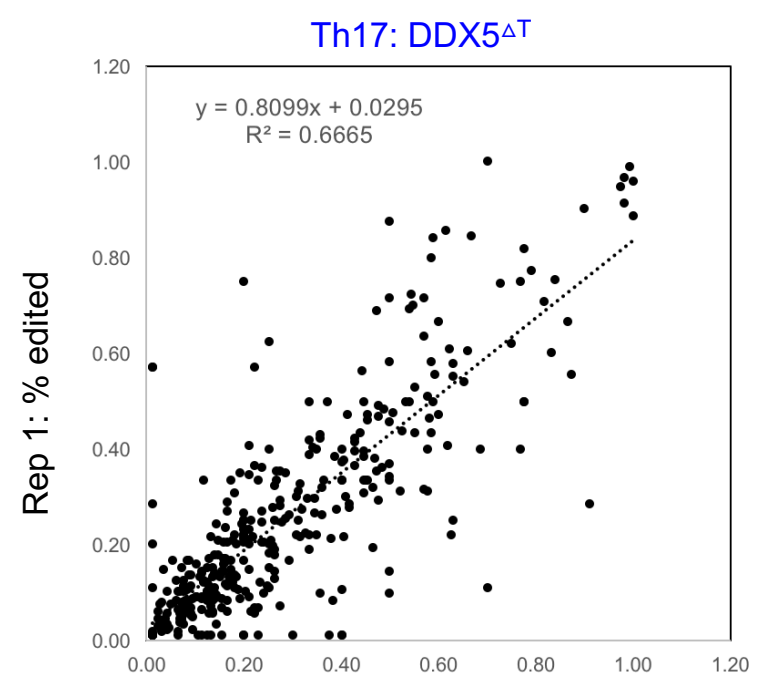

Rep 2: \% edited

Apobec3

\begin{tabular}{|c|c|}
\hline RNAseq & A-to-I Editing \\
\hline WT $^{\top}$ rep1 & 15.6 \\
\hline DDX5 ${ }^{\Delta \top}$ rep1 & 7.3 \\
\hline WT $^{\top}$ rep2 & 11.4 \\
\hline DDX5 $5^{\Delta}$ rep2 & 4.4 \\
\hline
\end{tabular}

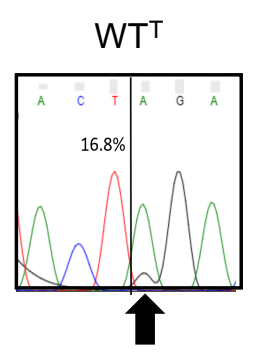

DDX5 ${ }^{\Delta T}$

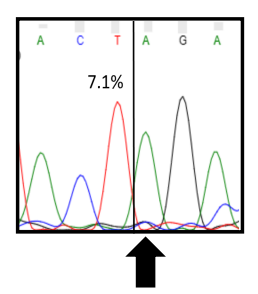

Apobec3

\begin{tabular}{|c|c|}
\hline Sanger & A-to-I Editing \\
\hline $\mathrm{WT}^{\mathrm{T}}$ rep3 & 16.8 \\
\hline $\mathrm{DDX} 5^{\Delta \mathrm{T}}$ rep3 & 7.1 \\
\hline $\mathrm{WT}^{\mathrm{T}}$ rep4 & 22.9 \\
\hline $\mathrm{DDX}^{\mathrm{s}} \mathrm{T}^{\mathrm{T}}$ rep4 & 9.3 \\
\hline
\end{tabular}




\section{Graphic Abstract}

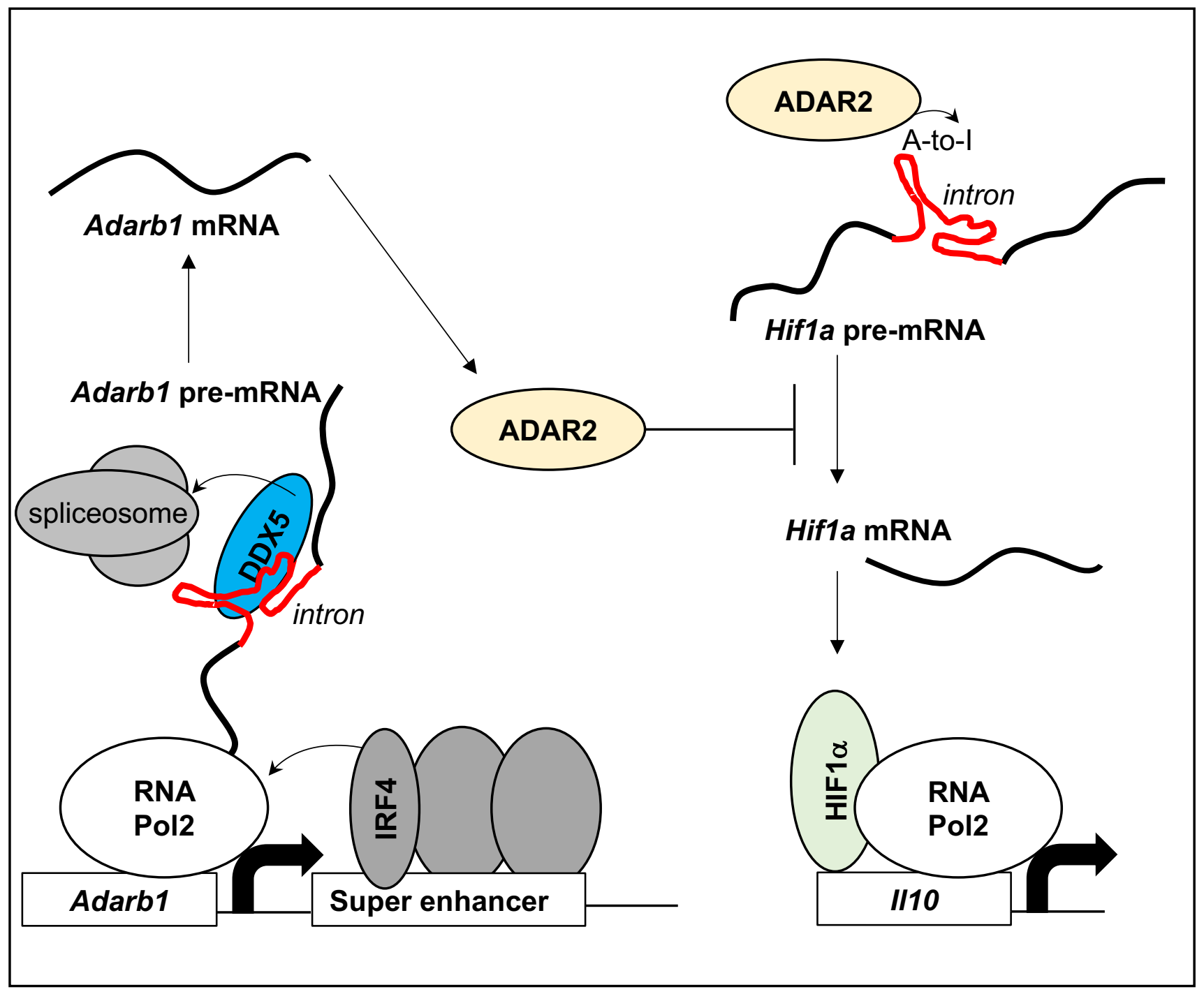

Major findings:

Naïve and Th17 cells harbored overlapping and unique A-to-I editome.

ADAR2 expression is induced during Th17 polarization under the control of an intragenic super enhancer.

ADAR2 targets Hif1a transcripts to regulate HIF1 $\alpha$ expression and IL10 production in $\mathrm{ROR} \mathrm{t}^{+}$Th17 cells in the small intestine.

Post-transcriptionally, Adarb1 pre-mRNAs are bound and regulated by RNA binding DEAD-box protein DDX5. DDX5-deficient Th17 cells have a dysregulated RNA editome and altered effector function. 